“C 2019 IEEE. Personal use of this material is permitted. Permission from IEEE must be obtained for all other uses, in any current or future media, including reprinting/republishing this material for advertising or promotional purposes, creating new collective works, for resale or redistribution to servers or lists, or reuse of any copyrighted component of this work in other works." 


\title{
Continuous Beam Scanning at a Fixed Frequency with a Composite Right/Left-Handed Leaky-Wave Antenna Operating Over a Wide Frequency Band
}

\author{
Shu-Lin Chen, Student Member, IEEE, Debabrata K. Karmokar, Member, IEEE, Zheng Li, Member, IEEE, \\ Pei-Yuan Qin, Member, IEEE, Richard W. Ziolkowski, Fellow, IEEE, and Y. Jay Guo, Fellow, IEEE .
}

\begin{abstract}
Fixed-frequency beam scanning leaky-wave antennas (LWAs) that can scan their main beam over a specific frequency band are highly desired for future wireless communication systems. A composite right/left-handed (CRLH) LWA is developed in this paper that facilitates fixed-frequency continuous beam scanning over a wide operational frequency band. A variation of a simple single-layer non-reconfigurable frequencybased continuous beam scanning CRLH LWA is considered first. Its dispersion properties are approximately investigated using an equivalent circuit model. It is reported how two groups of varactor diodes can be incorporated into its basic circuit model to electronically control its dispersion behavior. An optimized reconfigurable CRLH LWA with practical DC biasing lines is then realized from this non-reconfigurable design. Fixedfrequency continuous beam scanning, from backward to forward directions through broadside, is reported over a wide operational frequency band. Simulations predict that the antenna can operate from 4.75 to $5.25 \mathrm{GHz}$ with the main beam being continuously scannable at each frequency point. A prototype was fabricated, assembled, and tested. The measured results confirm its simulated performance characteristics.
\end{abstract}

Index Terms-Beam scanning, composite right/left-handed (CRLH), fixed-frequency, leaky-wave antenna (LWA), reconfigurable antenna.

\section{INTRODUCTION}

$\mathbf{L}$ EAKY-wave antennas (LWAs) are highly promising candidates to meet the demands for future wireless communication systems, including low cost, easy integration, and multiple functionalities. Generally, focused scanning beams are formed in an open, far-field region from power leaked serially from a guided wave structure. LWAs have substantial known benefits, e.g., they are low profile, have simple feed networks, and achieve wide-angle beam scanning capabilities [1]-[7]. Since the introduction of LWAs in the 1940s, two

Manuscript received XXX. This work was supported by the Australian Research Council (ARC) Discovery Project (DP) 160102219 grant, the Discovery Early Career Researcher Award (DECRA) DE170101203 grant, and the National Natural Science Foundation of China under Grant 61771038. (Corresponding author: Debabrata K. Karmokar.)

S.-L. Chen, P.-Y. Qin, R. W. Ziolkowski, and Y. J. Guo are with the Global Big Data Technologies Centre (GBDTC), University of Technology Sydney (UTS), Ultimo, NSW 2007, Australia (E-mail: Shulin.Chen@uts.edu.au).

D. K. Karmokar was with the Global Big Data Technologies Center (GBDTC), University of Technology Sydney (UTS), Ultimo, NSW 2007, Australia. He is now with the School of Engineering, Faculty of Science and Engineering, Macquarie University, Sydney, NSW 2109, Australia (e-mail: dkkarmokar@ieee.org).

$\mathrm{Z}$. Li is with the Institute of Lightwave Technology, Beijing Jiaotong University, Beijing 100044, China (E-mail: lizheng@bjtu.edu.cn) challenging, yet important issues have drawn great interest from the antenna community to advance their performance and, hence, to further enhance their desirability.

One has been the open stopband (OSB) problem, i.e., the realized gain usually significantly drops when the main beam is scanned through the broadside direction [8]-[10]. Two popular techniques have been developed in the recent two decades to suppress the OSB. One is to utilize a composite right/lefthanded (CRLH) structure [11]-[14]. Another approach is to excite a higher order harmonic of a periodic LWA, e.g., the $n=-1$ space harmonic, with impedance matching or reflection cancellation methods [15]-[19]. The second issue is that the beam scanning capability of LWAs is frequencybased rather than occurring at a fixed-frequency. Due to the dependency of the phase constant and the wave number on the source frequency, one needs to sweep it to scan the main angle.

Most LWAs reported to date that can scan their main beam are frequency-based. On the other hand, only a few fixed-frequency beam scanning LWAs have been realized. Prototypes have used microstrip, Fabry-Perot, and active metasurface structures [20]-[23]. However, these systems can only scan the beam in a quadrant (left or right quadrant) rather than across the full hemisphere from backward to forward through broadside. Most wireless communication systems prefer to transmit/receive data at a specific operating frequency within an allocated band, as well as to have continuous beam scanning at all frequencies within it [24]. Nonetheless, it is difficult to achieve this valuable fixed-frequency continuous backward to forward beam scanning through broadside. To the best of the authors' knowledge, only a few interesting solutions have been reported [25]-[33]. Continuous beam scanning from $-25^{\circ}$ to $+25^{\circ}$ was obtained in [25] by centrally feeding two independently controlled high impedance surfaces of a FabryPerot LWA. A reconfigurable partially reflective surface (PRS) antenna was reported in [28] to accomplish fixed-frequency beam scanning at the beam angles: $-15^{\circ}, 0^{\circ}$, and $+15^{\circ}$. A dielectric grating-based graphene leaky-wave antenna was developed in [29] to achieve a simulated beam scan from $-35.2^{\circ}$ to $+21.1^{\circ}$ at $1.5 \mathrm{THz}$ by varying the chemical potentials in a graphene surface, and hence, its plasmon polariton properties. A $45^{\circ}$ beam scan at a fixed operating frequency between 5.5 and $5.8 \mathrm{GHz}$ was achieved in [26] by using a modulated corrugated microstrip line. A microstrip line LWA with a reconfigurable 2-bit phase unit was developed in [27] that 
radiates five switchable beams at $5.0 \mathrm{GHz}$ and five prototypes were fabricated to validate these five working states.

Reconfigurable CRLH structures have realized fixedfrequency beam scanning [30]-[33]. They can exhibit an inherent continuous transition between their $\mathrm{RH}$ and $\mathrm{LH}$ dispersion curves when they are designed in their balanced condition. A reconfigurable CRLH LWA that continuously scanned its beam from $-13^{\circ}$ to $+18^{\circ}$ was realized in a recent work [32] by tuning the permittivity of a liquid crystal. Nevertheless, these reported fixed-frequency continuous beam scanning LWAs again have a limited scanning range, e.g., less than $60^{\circ}$. Moreover, only a few of them can realize fixedfrequency beam scanning over a band of frequencies and that band is narrow. Furthermore, if one would like to introduce switchable devices to achieve different phase constant states, it is challenging to isolate the polarities of their DC sources as well as to integrate practical DC biasing lines into their overall structure [27], [31]. These difficulties arise because LWAs with a simple feed structure generally have their radiators connected to a ground plane through a number of shorting vias.

A CRLH LWA is developed in this paper that realizes continuous beam scanning at a fixed-frequency over a wide operating frequency band. It is a simple configuration that has a single feed and incorporates practical DC biasing lines. Simulations predict that the antenna can achieve fixedfrequency continuous beam scanning over a $100^{\circ}$ range from 4.75 and $5.25 \mathrm{GHz}$, i.e., a $10 \%$ factional bandwidth relative to its center frequency, $5.0 \mathrm{GHz}$. An optimized prototype was fabricated, assembled, and measured. The theory of operation is described in Sections II and III. Its fixed-frequency scanning ability is illustrated in Section IV. The measured results are described and compared with previously reported systems in Section V. As noted in Section VI, there are several important contributions to the state-of-the-art reported. They include the development of a simple single-layer non-reconfigurable frequency-based continuous beam scanning CRLH LWA from a variation of the corresponding classical CRLH T-type circuit. They also include an in-depth analysis of the ability to tune its dispersion features through modifications of its effective circuit parameters and the development of a simple, yet effective DC biasing network which facilitates making this LWA reconfigurable. Finally, it is clearly demonstrated that the final optimized antenna system and its measured prototype realize continuous beam scanning at a fixed-frequency over a wide operating band while remaining a simple and costeffective configuration.

\section{THEORY}

A classical T-type equivalent circuit of a 1-D CRLH structure is depicted in Fig. 1. The inductance $L_{R}$ and capacitance $C_{R}$ are naturally associated with a conventional right-handed (RH) transmission line properties. In contrast, the placement of the inductance $L_{L}$ and capacitance $C_{L}$ lead to the left-handed (LH) transmission line properties [34]-[36]. The inductance $L_{L}$ in a microstrip version is typically introduced by shorting a via between the line and its ground plane. Similarly, the capacitance $C_{L}$ is introduced in the transmission line as a

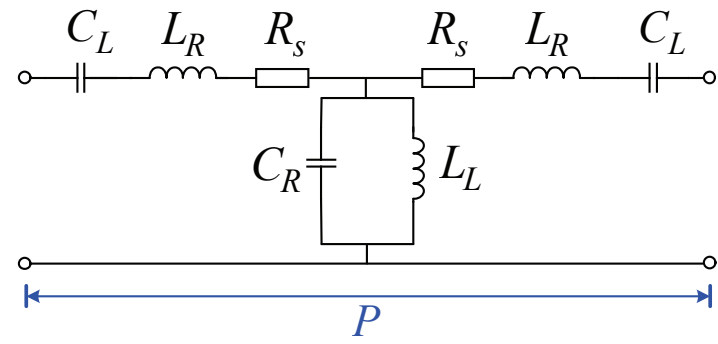

Fig. 1. T-type equivalent circuit of a 1-D conventional CRLH unit cell.

series of gaps or as an interdigitated-capacitor. The resistor $R_{s}$ denotes radiation losses from the unit cell.

Given $\gamma=\alpha+j \beta$ is the complex propagation constant, where $\alpha$ and $\beta$ are its attenuation constant and phase constant, respectively, one can obtain [34], [37]:

$$
\begin{aligned}
& \alpha=\frac{1}{P} \mathbf{I m}\left[\cos ^{-1}(1+\mathrm{ZY})\right] \\
& \beta=\frac{1}{P} \mathbf{R e}\left[\cos ^{-1}(1+\mathrm{ZY})\right]
\end{aligned}
$$

with $Z=R_{s}+j \omega L_{R}+1 /\left(j \omega C_{L}\right)$ and $Y=j \omega C_{R}+$ $1 /\left(j \omega L_{L}\right) . \omega$ is the angular frequency, i.e., $\omega=2 \pi f$, and $P$ is the length of the unit cell.

The main angle, $\theta$, of the beam radiated by a conventional frequency-based LWA at its source frequency $f$ is determined by the relation [38], [39]:

$$
\theta=\sin ^{-1}\left(\beta / k_{0}\right)
$$

where $\theta$ is defined as the angle measured from the broadside direction and $k_{0}$ is the wave number in air. It is known that the phase constant of a low-loss CRLH unit is approximately equal to that of a loss-less CRLH unit cell [34]. Consequently, the phase constant of the lossless CRLH unit cell was adopted for our analysis. It is expressed as:

$$
\beta=\frac{1}{P} \cos ^{-1}\left(1-\omega^{2} L_{R} C_{R}-\frac{1}{\omega^{2} C_{L} L_{L}}+\frac{C_{R}}{C_{L}}+\frac{L_{R}}{L_{L}}\right)
$$

The cut-off frequencies $f_{1}$ and $f_{2}$ of this circuit occur when $\beta=0$ for both the LH and RH dispersion curves. They are calculated as:

$$
f_{1}=\frac{1}{2 \pi \sqrt{L_{R} C_{L}}}, \quad \text { and } \quad f_{2}=\frac{1}{2 \pi \sqrt{L_{L} C_{R}}}
$$

It is now well-known that the balanced condition can be achieved if $f_{1}=f_{2}$, that gives $L_{R} / C_{R}=L_{L} / C_{L}$ [11].

As noted previously, (3) tells us that the main beam angle can be scanned at a fixed frequency if the values of the phase constant $\beta$ can be varied in a independent manner. To alter the phase constant at a specific frequency, (4) indicates that the only choice is to change the circuit parameters if the period length $P$ is fixed, i.e., to change the values of $\widetilde{L}_{L}, \widetilde{C}_{L}, \widetilde{L}_{R}, \widetilde{C}_{R}$ in some self-consistent manner. This is clarified by expressing the phase constant $\widetilde{\beta}$ at a specific frequency $f_{0}$ as:

$$
\widetilde{\beta}=\frac{1}{P} \cos ^{-1}\left(1-\omega_{0}^{2} \widetilde{L}_{R} \widetilde{C}_{R}-\frac{1}{\omega_{0}^{2} \widetilde{C}_{L} \widetilde{L}_{L}}+\frac{\widetilde{C}_{R}}{\widetilde{C}_{L}}+\frac{\widetilde{L}_{R}}{\widetilde{L}_{L}}\right)
$$




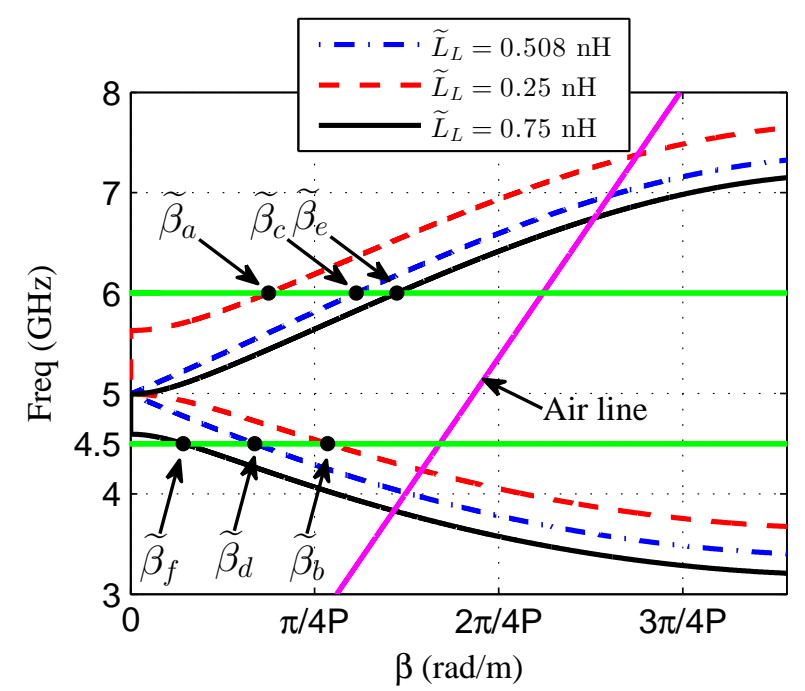

Fig. 2. Dispersion diagrams for different values of $\widetilde{L}_{L}$ in the equivalent CRLH circuit.

where $\omega_{0}=2 \pi f_{0}$, and consequently, the main beam angle $\tilde{\theta}$ as:

$$
\widetilde{\theta}=\sin ^{-1}\left(\widetilde{\beta} / k_{0}\left(f_{0}\right)\right)
$$

where $k_{0}\left(f_{0}\right)$ is the free space wave number at $f_{0}$. It is then clear that fixed-frequency beam scanning can occur if some combination of the four CRLH circuit parameters can be controlled to alter the phase constant $\widetilde{\beta}$. We have elected to change those parameters and, hence, the values of $\beta$ by introducing a set of switching devices into the circuit, e.g., a set of PIN diodes or varactor diodes, and by controlling them electronically.

To illustrate the concept, the values for the circuit parameters and the period length are chosen to be: $\widetilde{L}_{R}=1.6 \mathrm{nH}$, $\widetilde{C}_{R}=2 \mathrm{pF}, \widetilde{L}_{L}=0.508 \mathrm{nH}, \widetilde{C}_{L}=0.635 \mathrm{pF}$, and $P=14$ $\mathrm{mm}$. When the value of a specific parameter is changed, the other values remain fixed in this initial study. Fig.2 shows the dispersion diagrams for different values of $\widetilde{L}_{L}$. One observes that when $\widetilde{L}_{L}=0.508 \mathrm{nH}$, a continuous transition at $5.0 \mathrm{GHz}$ is obtained without any gap between the left-handed (LH) and right-handed (RH) portions. Thus, the equivalent CRLH LWA would radiate a broadside beam. On one hand, when $\widetilde{L}_{L}$ is reduced from 0.508 to $0.25 \mathrm{nH}$, most of both the $\mathrm{RH}$ and $\mathrm{LH}$ portions of the dispersion curves shift up in frequency. On the other, when $\widetilde{L}_{L}$ increases from 0.508 to $0.75 \mathrm{nH}$, most of both shift down. However, notice that as that inductance increases (decreases), the cut-off frequency for the LH (RH) portion of the gap remains the same while the gap's upper (lower) cut-off frequency increases (decreases) to $5.6(4.6) \mathrm{GHz}$ through the frequency translation of the RH (LH) portion.

From these dispersion diagrams, one finds at $6.0 \mathrm{GHz}$ that continuous fixed-frequency beam scanning will occur only in the forward quadrant as the value of $\widetilde{\beta}$ increases from $\widetilde{\beta}_{a}$ $\left(\widetilde{L}_{L}=0.25 \mathrm{nH}\right)$ to $\widetilde{\beta}_{c}\left(\widetilde{L}_{L}=0.75 \mathrm{nH}\right)$. Similarly, continuous fixed-frequency beam scanning will occur at $4.5 \mathrm{GHz}$ only in the backward quadrant as $\widetilde{\beta}$ increases from $\widetilde{\beta}_{f}\left(\widetilde{L}_{L}=\right.$ $0.75 \mathrm{nH})$ to $\widetilde{\beta}_{b}\left(\widetilde{L}_{L}=0.25 \mathrm{nH}\right)$. It can then be concluded that

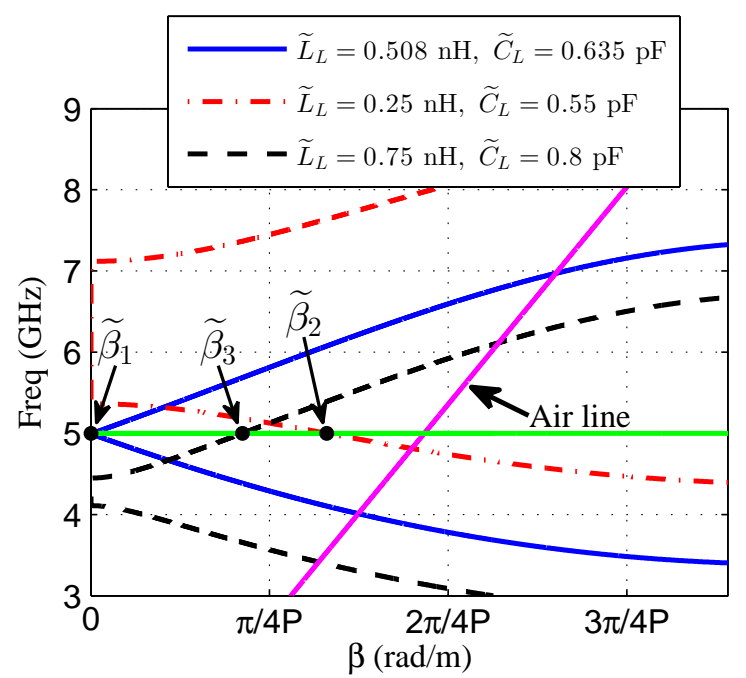

Fig. 3. Dispersion diagrams for different values of $\widetilde{L}_{L}$ and $\widetilde{C}_{L}$ in the equivalent circuit.

continuous scanning through broadside cannot occur if only one circuit parameter is changed, e.g., $\widetilde{L}_{L}$. Consequently, the effects of simultaneously changing two circuit parameters on the dispersion performance must be investigated.

Fig. 3 shows the different dispersion diagrams when the values of $\widetilde{L}_{L}$ and $\widetilde{C}_{L}$ are varied at the same time. We fix the antenna's operating frequency at $5.0 \mathrm{GHz}$. When $\left(\widetilde{L}_{L}, \widetilde{C}_{L}\right)=$ $(0.508 \mathrm{nH}, 0.635 \mathrm{pF}), \widehat{\beta}_{1}=0$ and broadside radiation is realized. On the other hand, when $\left(\widetilde{L}_{L}, \widetilde{C}_{L}\right)$ decrease separately to $(0.25 \mathrm{nH}, 0.55 \mathrm{pF})$, both the $\mathrm{LH}$ and $\mathrm{RH}$ dispersion curves shift upwards in frequency. Their cut-off frequencies separate, increasing to 5.36 and $7.07 \mathrm{GHz}$, respectively; and the phase term becomes $\widetilde{\beta}_{2}$ at $5.0 \mathrm{GHz}$, realizing a backward main beam direction. On the other hand, when they are increased separately to $(0.75 \mathrm{nH}, 0.8 \mathrm{pF})$, both the $\mathrm{LH}$ and $\mathrm{RH}$ dispersion curves shift downwards in frequency. Their cut-off frequencies separate, decreasing to 4.45 and $4.11 \mathrm{GHz}$, respectively; and the phase term becomes $\widetilde{\beta}_{3}$ at $5.0 \mathrm{GHz}$, realizing a forward main beam direction. In this manner, it is realized that one can achieve continuous beam scanning from backward to forward through broadside at a fixed frequency by simultaneously changing the values of $\widetilde{L}_{L}$ and $C_{L}$.

Finally, the effects on the dispersion performance were also investigated when three circuit parameters were simultaneously changed. Fig. 4 shows the dispersion diagrams when the values of $\widetilde{L}_{L}, \widetilde{C}_{L}$, and $\widetilde{L}_{R}$ are varied simultaneously. When $\left(\widetilde{L}_{L}, \widetilde{C}_{L}, \widetilde{L}_{R}\right)=(0.508 \mathrm{nH}, 0.635 \mathrm{pF}, 1.6 \mathrm{nH})$, the beam points in the broadside direction at $5.0 \mathrm{GHz}$ with the phase factor $\widetilde{\beta}_{1}=0$. When all their values are decreased to $(0.35$ $\mathrm{nH}, 0.55 \mathrm{pF}, 1.3 \mathrm{nH}$ ), both the $\mathrm{LH}$ and $\mathrm{RH}$ dispersion curves shift upwards yielding $\widetilde{\beta}_{2}$ and a backward beam direction at $5.0 \mathrm{GHz}$. On the other hand, when $\widetilde{L}_{L}$ and $\widetilde{C}_{L}$ are increased to $(1.3 \mathrm{nH}, 1.1 \mathrm{pF})$, while the value of $\widetilde{L}_{R}$ is further reduced to $1.1 \mathrm{nH}$, one then achieves $\widetilde{\beta}_{3}$ and a forward beam direction at $5.0 \mathrm{GHz}$. Thus, one finds that varying three circuit parameters simultaneously, more flexibility in controlling the beam direction is realized. However, it is also recognized that 


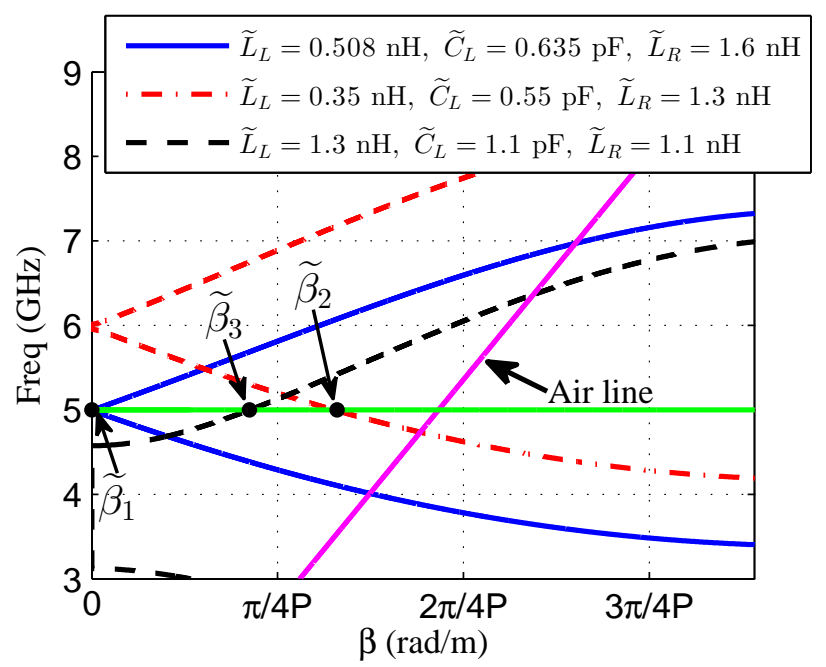

Fig. 4. Dispersion diagrams for different values of $\widetilde{L}_{L}, \widetilde{C}_{L}$, and $\widetilde{L}_{R}$ in the equivalent circuit.

this flexibility would come at the cost of an increased number of the switching elements and the associated complexity of their biasing network.

Several guidelines to change the circuit parameters $\left(\widetilde{L}_{L}, \widetilde{C}_{L}, \widetilde{L}_{R}, \widetilde{C}_{R}\right)$ and, hence, to alter the disperson diagrams in a controllable manner follow from these parameter studies. They are summarized as follows:

1) The values of the phase constant at a specific frequency can be varied by changing one or a combination of the circuit parameters $\left(\widetilde{L}_{L}, \widetilde{C}_{L}, \widetilde{L}_{R}, \widetilde{C}_{R}\right)$. The main beam can then be scanned at a fixed frequency.

2) If only one parameter value is changed while the other three parameters remain fixed, beam scanning at a fixed operating frequency is limited either to the forward or backward quadrants.

3) When combinations of two to four parameters values are changed simultaneously, the main beam can be continuously scanned at a specific frequency from the backward to the forward quadrant through broadside. There is a tradeoff between flexibility and complexity when the number of tunable parameters increases.

4) Generally, the introduced LH parameters, $\widetilde{\sim}_{L}$ and $\widetilde{C}_{L}$, are easier to modify than their RH ones, $\widetilde{L}_{R}$ and $\widetilde{C}_{R}$, because the latter arise naturally from the transmission line.

Based on these guidelines, we elected to control the two LH parameters, $\widetilde{L}_{L}$ and $\widetilde{C}_{L}$, to facilitate realizing a fixedfrequency beam scanning LWA.

\section{Frequency-Based Continuous Beam Scanning CRLH LWA}

A simple single-layer frequency-based continuous beam scanning CRLH LWA is developed first. It is shown in Fig. 5 and follows from the T-type equivalent circuit shown in Fig. 1. A two-layer copper-clad substrate is utilized. It has the following characteristics. It has the permittivity $\left(\varepsilon_{r}\right)$ is 2.2. Its loss tangent $(\tan \delta)$ is 0.001 . Its height, width, and

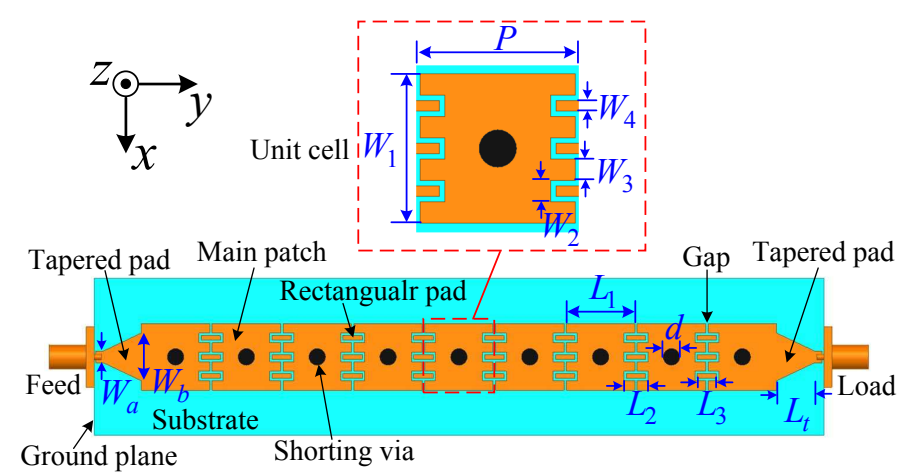

Fig. 5. Configuration of a single-layer frequency-based continuous beam scanning CRLH LWA. The top inset represents a unit cell of the antenna.

TABLE I

Optimized Design Parameter Values (in Millimeters) OF the FREQUENCY-BASED CONTINUOUS BEAM SCANNING CRLH LWA

\begin{tabular}{ccccccc}
\hline \hline Parameters & $L_{1}$ & $L_{2}$ & $L_{3}$ & $W_{1}$ & $W_{2}$ & $W_{3}$ \\
\hline Values $(\mathrm{mm})$ & 14.5 & 5.1 & 4.1 & 14 & 2 & 2 \\
\hline \hline Parameters & $W_{4}$ & $P$ & $d$ & $W_{a}$ & $W_{b}$ & $L_{t}$ \\
\hline Values $(\mathrm{mm})$ & 1 & 14.7 & 3.5 & 2.5 & 10 & 8 \\
\hline \hline
\end{tabular}

length are, respectively, $1.575 \mathrm{~mm}, 33 \mathrm{~mm}$, and $154.5 \mathrm{~mm}$. The main patches, rectangular pads and tapered pads are printed on the top layer. The bottom acts as a solid ground plane. This antenna consists of nine unit cells.

The configuration of each unit cell is shown in the top inset in Fig. 5. Its main patch is connected to the solid ground plane with a centrally located shorting via. Meander gaps are etched at both ends of this main patch. Rectangular pads are inserted into the meander gaps. This unit cell evolved naturally from a conventional RH microstrip transmission line. Two widely-used yet simple and effective methods were employed to introduce its LH properties, i.e., the center shorting vias produce the LH inductance and the meander gaps yield the LH capacitance.

The length and width of the patch are $L_{1}$ and $W_{1}$, respectively. The diameter of the shorting vias is $d$. The dimensions of the meander gap and the rectangular pads are represented by $L_{2}, L_{3}, W_{2}, W_{3}, W_{4}$. The length of the unit cell is $P$. It represents the length of one period of the LWA. The antenna is fed by a $50 \Omega$ source from the left and terminated in a 50 $\Omega$ load on the right. Tapered pads are symmetrically printed at both ends of the antenna. They provide impedance matching of the feed and load SMAs to the central set of unit cells over a wide frequency bandwidth. The dimensions of the tapered pad are $W_{a}, W_{b}$, and $L_{t}$.

To obtain the requisite values of the capacitances and inductances to achieve the balanced condition, the preliminary dimensions of the antenna's unit cell were selected and calculated using the microstrip equations for the capacitance and inductance given in [40], [41]. The complete antenna is shown in Fig. 5. Its unit cells and matching elements were analysed, simulated, and optimized with the ANSYS high- 


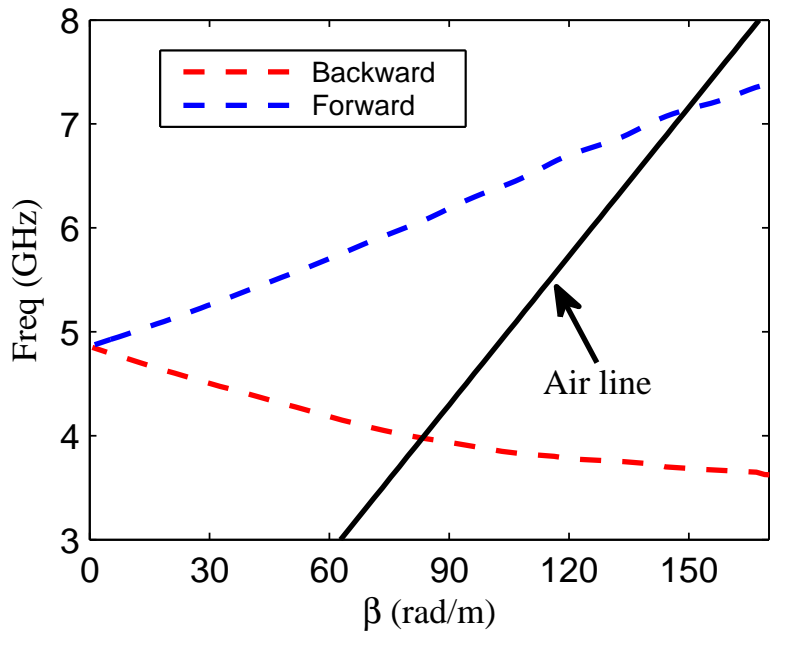

Fig. 6. Dispersion diagrams of the unit cell of the frequency-based continuous beam scanning CRLH LWA.

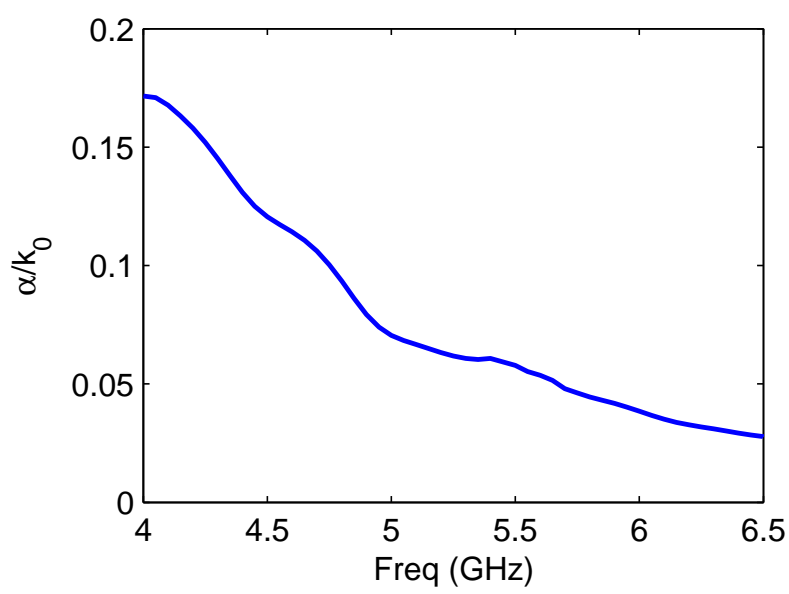

Fig. 7. Normalized attenuation constant of the unit cell of the frequency-based continuous beam scanning CRLH LWA.

frequency structure simulator (HFSS). The optimized values (in millimeters) of the antenna's design parameters are given in Table I. The phase constant and the attenuation constant of its unit cell are shown in Figs. 6 and 7, respectively. One can see from Fig. 6 that a continuous transition from the backward and to the forward dispersion curves occurs at $4.86 \mathrm{GHz}$, i.e., there is no bandgap. Furthermore, as observed from Fig. 7, the attenuation constant steadily decreases as the source frequency increases, and the values around the transition frequency are small but non-zero. These behaviors demonstrate that there is no OSB for this antenna and, hence, it can continuously scan the main beam from the backward to the forward directions through broadside [42], [43].

Fig. 8 shows the corresponding simulated S-parameters as functions of the operating frequency. The simulated $\left|S_{11}\right| \leq$ $-10 \mathrm{~dB}$ bandwidth is determined to be $2.01 \mathrm{GHz}$, from 4.02 to $6.03 \mathrm{GHz}$. The values actually remain below $-8.8 \mathrm{~dB}$ down to $4 \mathrm{GHz}$ and below $-5.8 \mathrm{~dB}$ up to $6.5 \mathrm{GHz}$. The values of $\left|S_{21}\right|$ increase stably with the operating frequency. Their peak

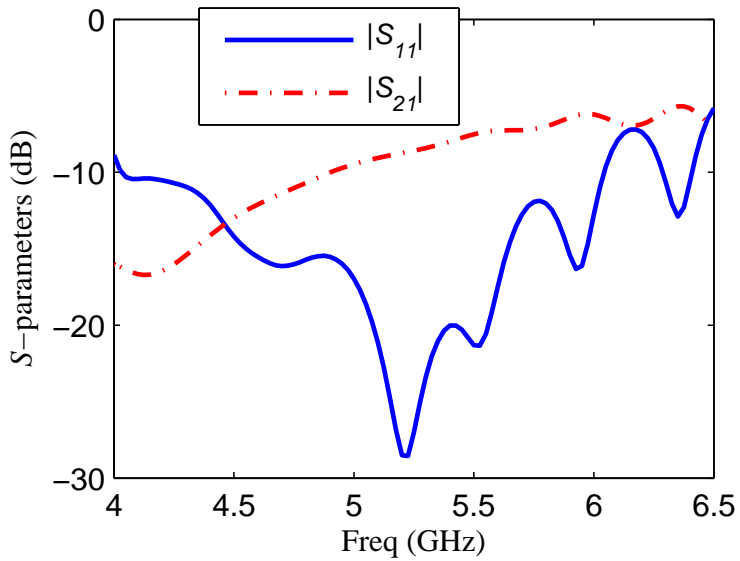

Fig. 8. Simulated S-parameters of the frequency-based continuous beam scanning CRLH LWA.

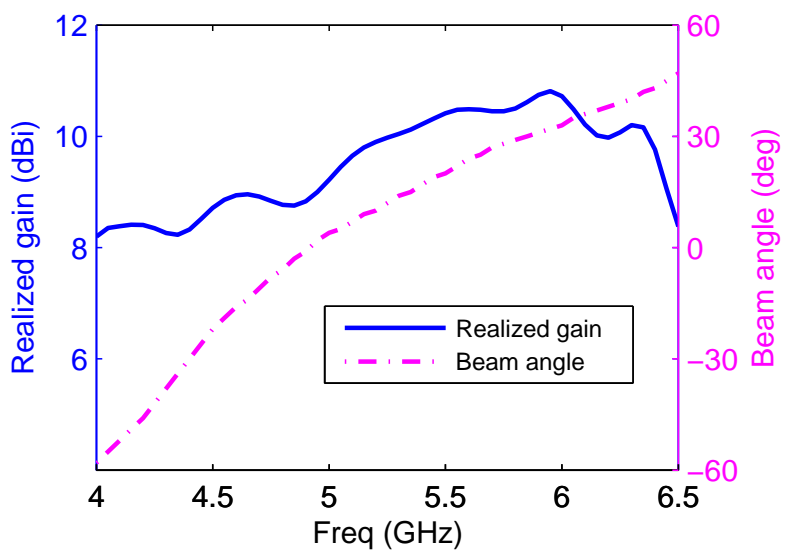

Fig. 9. Simulated realized gain and beam angle values of the frequency-based continuous beam scanning CRLH LWA.

value, $-5.7 \mathrm{~dB}$, occurs at $6.35 \mathrm{GHz}$.

The fields radiated by the meander gaps between the adjacent unit cells are the main contributions to the antenna's farfield radiation performance. Thus, the co-polarized component of the electrical field in the YZ plane is $E_{\theta}$, and its crosspolarized component is $E_{\phi}$. Fig. 9 shows the simulated realized gain and beam angle values as functions of the operating frequency. The realized gain exhibits a stable performance with a variation only between $8.2 \mathrm{dBi}$ (at $4 \mathrm{GHz}$ ) and 10.8 $\mathrm{dBi}\left(\right.$ at $5.95 \mathrm{GHz}$ ). The main beam angle varies from $-58^{\circ}$ to $+47^{\circ}$ when the frequency sweeps from 4 to $6.5 \mathrm{GHz}$. The broadside radiation occurs at $4.9 \mathrm{GHz}$ with a realized gain of $8.8 \mathrm{dBi}$.

\section{Fixed-Frequency Continuous Beam Scanning CRLH LWA}

A realizable fixed-frequency beam scanning CRLH LWA emerged from this simple design. Varactor diode switches were introduced to change the CRLH parameters and, hence, to reconfigure the dispersion diagrams for each operating point. A practical layout strategy for the DC biasing lines associated with these switches was also developed. 


\section{A. Antenna Configuration}

The two circuit parameters, i.e., $\widetilde{L}_{L}$ and $\widetilde{C}_{L}$, were selected for reconfigurability. Two groups of varactor diodes are employed to realize this reconfiguration. Another substrate layer is incorporated into the design for printing their bias lines. This multi-layered configuration is illustrated in Fig. 10. It has three metal layers, i.e., the patch layer, ground layer, and biasing layer. The patch and ground layers were, respectively, printed on the top and bottom of the upper substrate. For the lower substrate, all of the metal on its top surface was removed, and the biasing layer was printed on its bottom face. The substrates are the same as the one used in the initial design. The height of the upper substrate and the lower substrate is $h_{a}$ and $h_{b}$, respectively. The other design parameters are the same as those in the initial configuration.

Figs. 11(a), (b) and (c) show, respectively the patch, ground, and biasing layers. The patches and rectangular pads in the meander gaps of the unit cells, traces that connect the rectangular pads between each unit cell, and the source and termination feedlines with their shorted-stubs are printed on the patch layer, the upper surface of the antenna. One group of the varactor diodes, $\mathrm{A}$, is placed between the central rectangular pads and the main patches. Because these meander gaps introduce the left-handed capacitances $\widetilde{C}_{L}$, this location of the varactor diodes allows reconfiguration of that capacitance. The varactor diodes $\mathrm{A}$ are biased through the trace connecting the three rectangular pads in each meander gap. Note that the directions of these A varactor diodes, which are placed at the two ends of each central rectangular pad, are reversed. In this manner, they can be biased simultaneously with a single DC source.

As shown in Fig. 11(b), square-ring-shaped slots are etched into the ground plane layer, i.e., the middle metal layer. Smaller square pads are centered in these slots and, consequently, are isolated from the ground plane. The dimensions of the square-ring-shaped slots and the square pads are represented by $L_{s}$ and $L_{e}$. Two varactor diodes B are then reversely soldered between each square pad and the ground plane. Their orientations are opposite to those on the upper layer. Shorting vias A are centrally located with respect to both the main patches on the upper layer and the square pads on the bottom layer. Consequently, the connections between the shorting vias A and the ground plane are controlled by the varactor diodes B. Thus, the left-handed inductance $\widetilde{L}_{L}$ is introduced when the shorting vias A are turned on. Furthermore, this inductance can be tuned by varying the capacitance of the varactor diodes B. Since the prototype antenna has nine unit cells, eighteen varactor diodes A and twenty varactor diodes B are employed for its frequency and beam reconfiguration.

As shown in Fig. 11(c), two groups of DC pads and two long DC biasing lines, DC \#1 and DC \#2, are printed on the biasing layer. The varactor diodes $\mathrm{B}$ are soldered to the ground layer. Because this soldering causes significant bumps, a continuous rectangular slot was cut out of the bottom layer between the DC bias lines in order to have it lie flat on the ground layer. Similar rectangular cuts were made at the ends of this layer to facilitate the placement of the feed and load

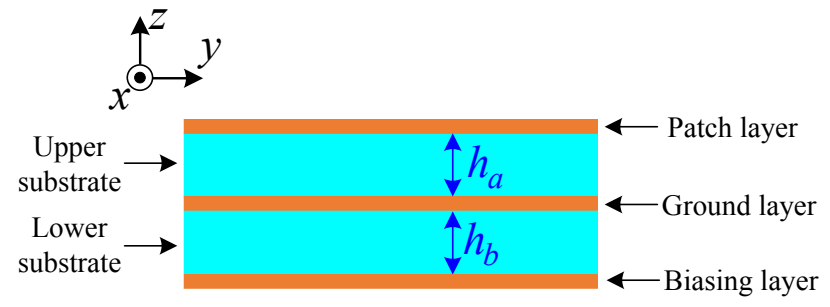

Fig. 10. Side view (not to scale) of the developed fixed-frequency beam scanning CRLH LWA.

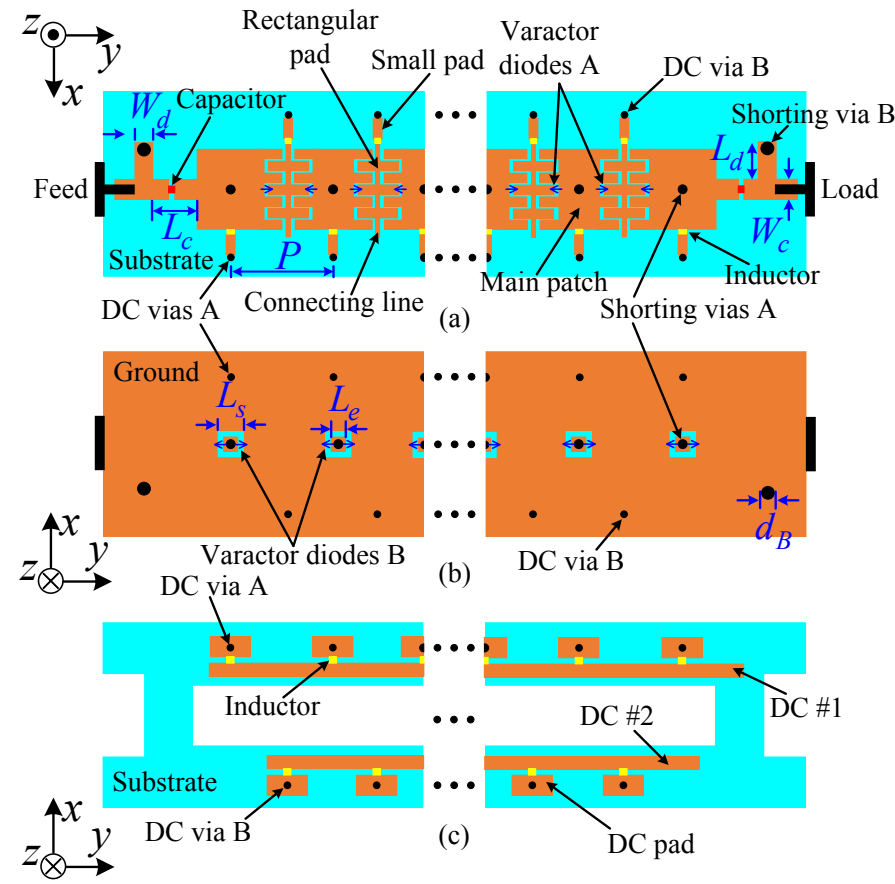

Fig. 11. The fixed-frequency beam scanning CRLH LWA configuration. (a) Top view of the patch layer. (b) Bottom view of the ground layer. (c) Bottom view of the biasing layer.

\section{SMA connectors.}

This reconfigurable antenna is again fed by a $50 \Omega$ source from its left side and terminated in $50 \Omega$ load on its right side. However, in contrast to the initial design, tapered termination pads are not employed for matching purposes. The microstrip structures illustrated in Fig. 11(a) are used instead. A shortedstub with a shorting via B is introduced into these terminating lines to facilitate the requisite impedance matching between these feedlines and the periodic portion of the antenna. The dimensions of the shorted-stub and the shorting via B are denoted by $L_{c}, W_{c}, L_{d}, W_{d}$ and $d_{B}$.

\section{B. DC Biasing Strategy}

For a clear understanding of the DC biasing network, a three-dimensional (3D) view of the entire system is depicted in Fig. 12 without the substrates being present. It required a careful design. Two capacitors are located between the feedlines and the first and last main patches to isolate the DC signals while maintaining the continuity of the RF signals. Inductors are employed to choke the RF signal while maintaining the continuity of the DC signal. The positive polarity of each of 


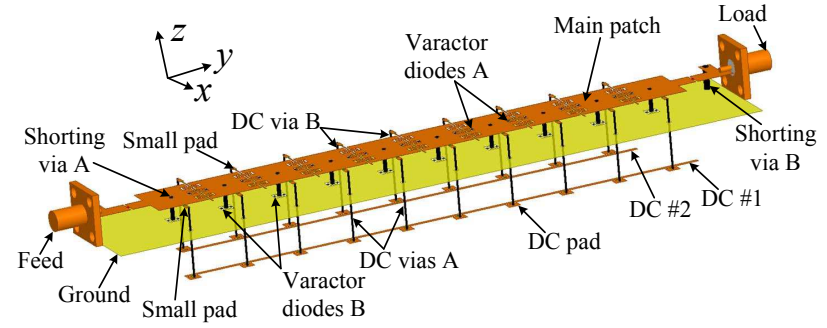

Fig. 12. A 3D view (not to scale) of the fixed-frequency beam scanning CRLH LWA without the substrates being present.

TABLE II

Design Parameter Values of THE Optimized FiXed-Frequency BEAM SCANNING CRLH LWA

\begin{tabular}{cccccccccc}
\hline \hline Parameters & $L_{1}$ & $L_{2}$ & $L_{3}$ & $W_{1}$ & $W_{2}$ & $W_{3}$ & $W_{4}$ & $P$ & $d$ \\
\hline Values $(\mathrm{mm})$ & 13 & 5 & 4 & 14 & 2 & 2 & 1 & 14 & 1 \\
\hline \hline Parameters & $L_{c}$ & $W_{c}$ & $L_{d}$ & $W_{d}$ & $L_{s}$ & $L_{e}$ & $d_{B}$ & $h_{a}$ & $h_{b}$ \\
\hline Values $(\mathrm{mm})$ & 9.75 & 4.5 & 5 & 3 & 3 & 2 & 1.5 & 1.575 & 0.8 \\
\hline \hline
\end{tabular}

the varactor diodes $\mathrm{A}$ on the patch layer is connected to a main patch. The negative polarity is connected to the rectangular pads in the meander gaps. The positive polarity of each of the varactor diodes $\mathrm{B}$ on the ground layer is connected to a small square pad, and then connected to a main patch through a shorting via $\mathrm{A}$. The negative polarity is connected directly to the ground plane. Note that the varactor diodes A and varactor diodes B share the same positive polarity.

A set of small rectangular strips are distributed on the patch layer. They are placed close to the outside (horizontal) edges of the main patches. Each one is connected to the main patch of a unit cell through an inductor. Furthermore, these small pads are also connected to the DC pads on the biasing layer through a DC via A. Each of the latter is connected to the bias line DC \#1 through an inductor. Thus, the positive polarity of each varactor diode $\mathrm{A}$ and also each varactor diode $\mathrm{B}$ is connected to DC \#1. Similarly, the connecting lines and, hence, the rectangular pads in the meander slots are all connected to the bias line DC \#2 through the small pads on the upper surface, the $\mathrm{DC}$ vias $\mathrm{B}$, and the $\mathrm{DC}$ pads on the bottom layer. Thus, the negative polarity of each varactor diode $\mathrm{A}$ is connected to DC \#2. Consequently, when a DC voltage is applied between the bias lines DC \#1 and DC \#2, all eighteen varactor diodes A can be biased simultaneously with a single DC source. When a DC voltage is applied between the bias lines DC \#1 and the ground plane, all twenty varactor diodes B are biased simultaneously. This DC biasing strategy was simple, yet it turned out to be very effective.

\section{Results And Discussion}

\section{A. Simulated Results}

The performance characteristics of the fixed-frequency beam scanning CRLH LWA were optimized with a series of HFSS simulations. The final design parameters are listed

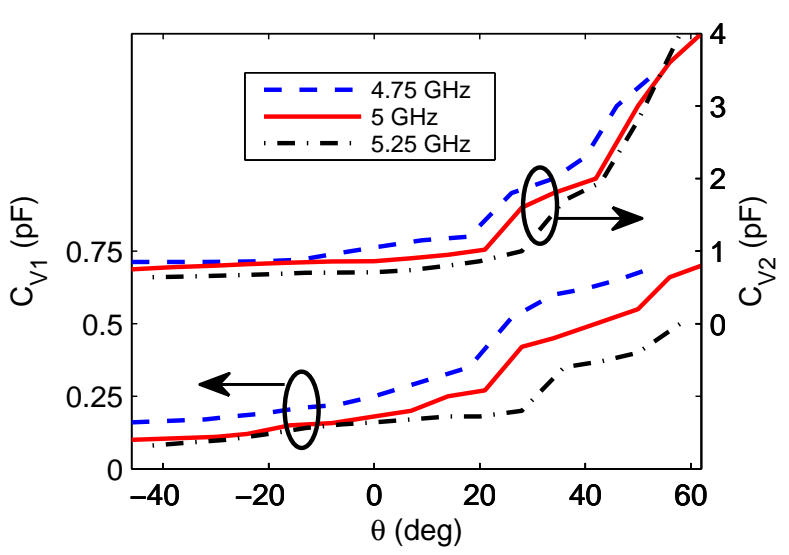

Fig. 13. Capacitance values of $C_{V 1}$ and $C_{V 2}$ as functions of the simulated main beam direction at $4.75,5$, and $5.25 \mathrm{GHz}$.

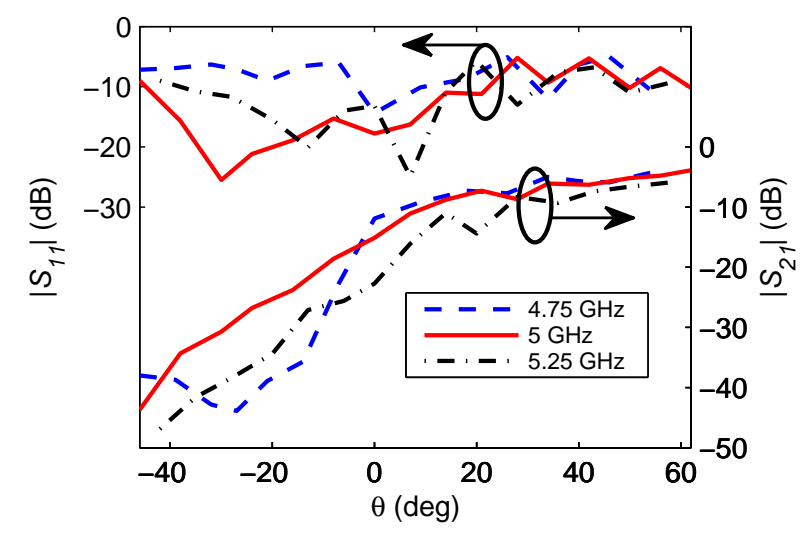

Fig. 14. S-parameters as functions of the simulated main beam direction at $4.75,5$, and $5.25 \mathrm{GHz}$.

in Table II. The simulation results indicated that this fixedfrequency beam scanning LWA would operate over a $10 \%$ frequency bandwidth, i.e., 4.75 to $5.25 \mathrm{GHz}$, and for each frequency point within the bandwidth, the main beam can be continuously scanned from the backward to forward directions through broadside.

As illustrations, the simulated performance of the optimized LWA working at $4.75,5$, and $5.25 \mathrm{GHz}$ is shown in Figs. 13-15. Fig. 13 shows the values of the capacitances $C_{V 1}$ and $C_{V 2}$ as functions of the simulated main beam direction. One notes that with increasing values of $\left(C_{V 1}, C_{V 2}\right)$, the main beam can be continuously scanned from $-46^{\circ}$ to $+54^{\circ}$, $-46^{\circ}$ to $+62^{\circ}$, and $-42^{\circ}$ to $+58^{\circ}$ at $4.75,5$, and $5.25 \mathrm{GHz}$, respectively. It is noticed that the slopes of these curves are relatively flat for the backward main beam angles and begin to sharply increase for the forward angles. This behavior indicates that the main beam angles in the backward direction are more sensitive to variations in the values of $C_{V 1}$ and $C_{V 2}$ as compared to the ones in the forward direction. Fig. 14 shows the simulated S-parameters as functions of the simulated main beam direction. It is seen that for most main beam angles, the values of $\left|S_{11}\right|$ are close to or below $-10 \mathrm{~dB}$, and the values of $\left|S_{21}\right|$ increase as the main beam scans from backward to forward direction. Simulations also indicate that the radiation 


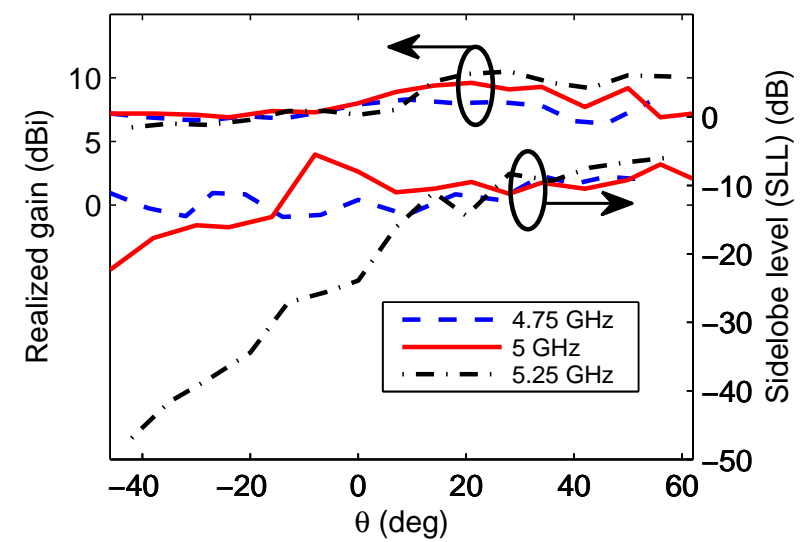

Fig. 15. Sidelobe level (SLL) and realized gain as functions of the simulated main beam direction at $4.75,5$, and $5.25 \mathrm{GHz}$.

efficiency decreases with the increase of the main beam angle. The radiation efficiency is over $90 \%$ for the backward main beam angles, and is more than $60 \%$ within the whole scanning range. The sidelobe level (SLL) and realized gain as functions of the simulated main beam direction at $4.75,5$, and $5.25 \mathrm{GHz}$ are shown in Fig. 15.

It is noted that the varactor diodes $\mathrm{A}$ and $\mathrm{B}$ were treated as lossless for these simulation studies, i.e., they were taken simply to be equivalent to variable capacitors with capacitances $C_{V 1}$ and $C_{V 2}$, respectively. No resistance values were included. This choice was made because of the number of diodes and their known manufacturing tolerances. Moreover, discrepancies were expected between their actual performance characteristics and the manufacturer's models, which were specified at a frequency much lower than the antenna's operating frequencies. Nonetheless, it was also anticipated that while the lossless models would predict gain values higher than the actual measured values, the average results of all the diodes would not cause a large difference between the predicted and measured beam angles and radiation patterns. These outcomes were confirmed with the testing of the prototype system.

The simulated radiation patterns for six operating states of the antenna, i.e., States $1-6$, at $5 \mathrm{GHz}$ are shown in Fig. 16 . It is noted that the half-power beamwidths (HPBWs) of the backward beams are wider than those of the forward beams. For example, the HPBW is $47^{\circ}$ for State $1\left(C_{V 1}, C_{V 2}\right)=(0.1$ $\mathrm{pF}, 0.75 \mathrm{pF})$ and is $24^{\circ}$ for State $5\left(C_{V 1}, C_{V 2}\right)=(0.45 \mathrm{pF}, 1.8$ $\mathrm{pF})$. This feature occurs because the radiation in the first few states (backward pointing) is generated primarily by only the first few unit cells of the LWA while most of them contribute to it for the latter few states (forward pointing). Fig. 17 shows the realized gain as a function of the frequency for the above six operating states at $5.0 \mathrm{GHz}$. One can observe that the antenna's 3-dB operating bandwidth increases as the main beam angle direction increases. This phenomenon is caused by the aforementioned sensitivity of the main beam angles to the values of the capacitances $C_{V 1}$ and $C_{V 2}$ as illustrated in Fig. 13.

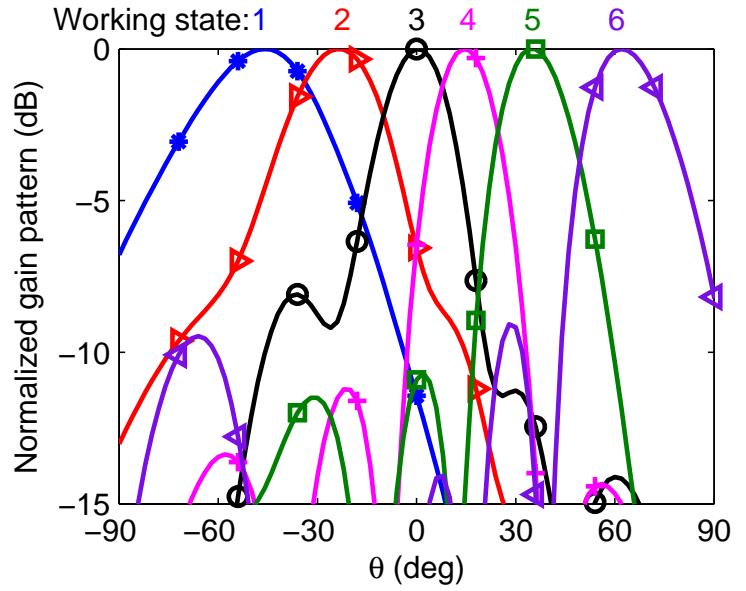

Fig. 16. Simulated radiation patterns for the antenna's six operating states at $5 \mathrm{GHz}$.

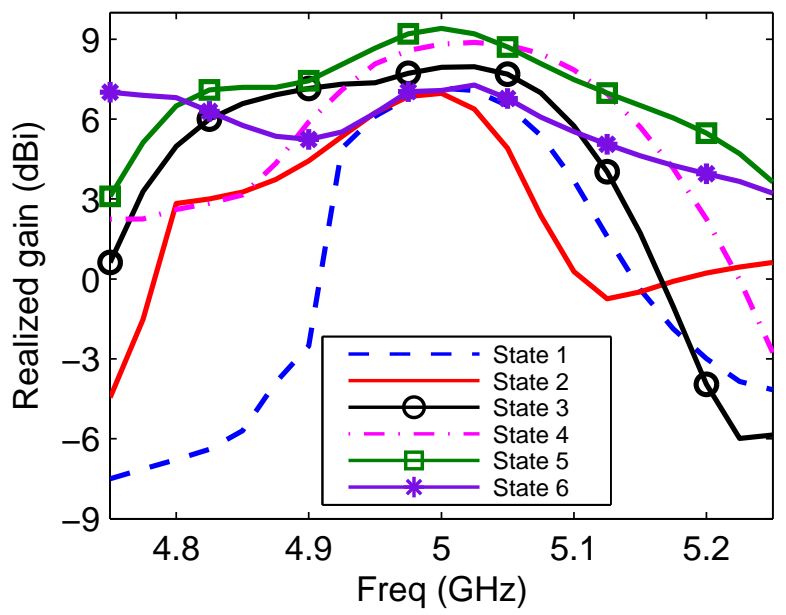

Fig. 17. Realized gain as a function of the frequency for six operating states of the antenna at $5 \mathrm{GHz}$.

\section{B. Measured Results}

This optimized design was fabricated and tested. Figs. 18(a) and (b) show photographs of this prototype, respectively, before and after assembly. Two types of commercial varactors were utilized to realize the capacitance tuning. A flip-chip varactor diode MA46H120 produced by MACOM company was used for the varactor diodes A. Its datasheet indicates that when the reverse biasing voltage is varied from 0 to 18 $\mathrm{V}$, the capacitance value is reduced from 1.3 to $0.15 \mathrm{pF}$. The associated series resistance is approximately $2 \Omega$ at $0.5 \mathrm{GHz}$ [44]. On the other hand, a silicon abrupt junction varactor diode SMV1405 from Skyworks company was used for the varactor diodes $\mathrm{B}$. Its datasheet indicates that when the reverse biasing voltage is varied from 0 to $30 \mathrm{~V}$, the capacitance value is reduced from 2.67 to $0.63 \mathrm{pF}$. The associated series resistance is smaller, approximately $0.8 \Omega$ at $0.5 \mathrm{GHz}$ [45]. Unfortunately, these were the lowest loss diodes that we could purchase. Their capacitance ranges fall short of the simulated values and only four $(5.25 \mathrm{GHz})$ and five $(5.0 \mathrm{GHz})$ of the 

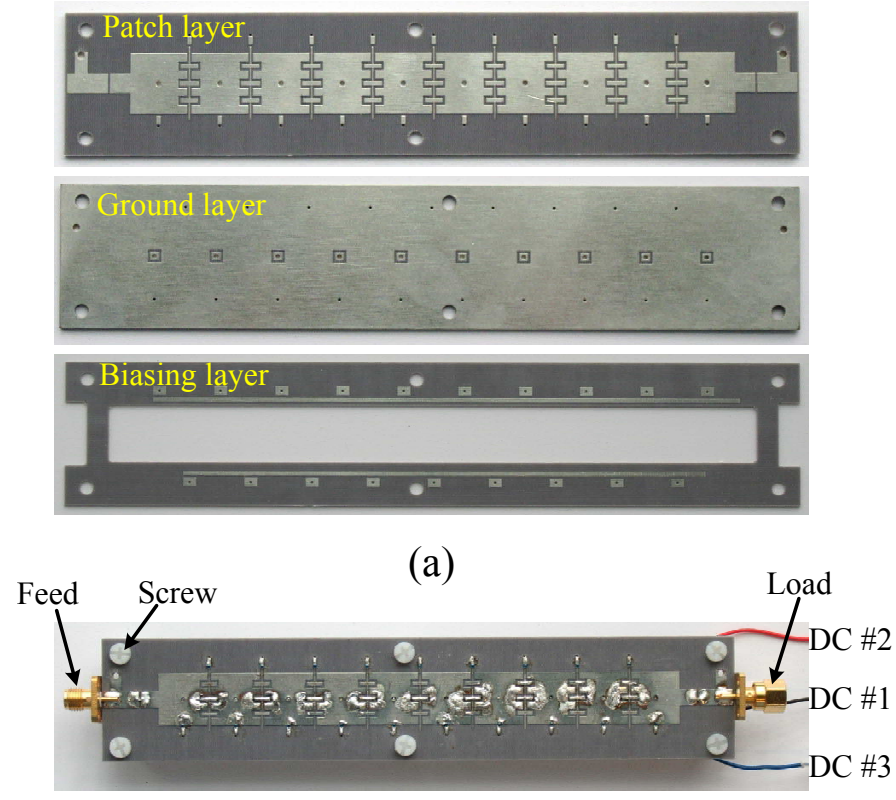

(b)

Fig. 18. Photographs of the fabricated fixed-frequency beam scanning CRLH LWA prototype. (a) Before assembly. (b) After assembly.

TABLE III

Measured Performance Characteristics of the FIXED-FREQUENCY BEAM SCANNING CRLH LWA OPERATING AT $5 \mathrm{GHZ}$

\begin{tabular}{c|ccccc}
\hline \hline \multirow{2}{*}{ Antenna performance } & \multicolumn{5}{|c}{ States } \\
\cline { 2 - 6 } & 1 & 2 & 3 & 4 & 5 \\
\hline Voltage $V_{1}(\mathrm{~V})$ & 18 & 18 & 10.5 & 3.1 & 2.4 \\
\hline Voltage $V_{2}(\mathrm{~V})$ & 16 & 13 & 11 & 2 & 0.1 \\
\hline$S_{11}(\mathrm{~dB})$ & -16.8 & -12.9 & -10.7 & -9.6 & -10.0 \\
\hline$S_{21}(\mathrm{~dB})$ & -39.9 & -34.7 & -35.3 & -18.3 & -20.7 \\
\hline Main beam angle & $-37^{\circ}$ & $-19^{\circ}$ & $0^{\circ}$ & $+24^{\circ}$ & $+32^{\circ}$ \\
\hline Realized gain $(\mathrm{dBi})$ & 5.7 & 4.9 & 5.4 & 5.7 & 4.6 \\
\hline \hline
\end{tabular}

six simulated states could be measured effectively. Every inductor was the surface-mount inductor 0402HP-8N7X (8.7 $\mathrm{nH})$ from Coilcraft. According to its datasheet and confirmed by communications with Coilcraft's technical support, it has a self-resonant frequency at $5.0 \mathrm{GHz}$ and sufficient bandwidth to choke the RF signals within the frequency band of interest while maintaining the DC continuity. Every DC signal blocking capacitor was selected to be the 600L-0402 capacitor from American Technical Ceramics (ATC) company with a value of $8.2 \mathrm{pF}$.

To verify the fixed-frequency beam scanning performance, the far-field radiation characteristics of the prototype were measured using the spherical near-field (SNF) antenna measurement system NSI-700S-50 located at CSIRO, Marsfield, Australia. Due to the limitations of this facility, measurements were only conducted at 5.0 and $5.25 \mathrm{GHz}$. Table III presents the measured performance of the fixed-frequency beam scanning CRLH LWA's five working states at $5.0 \mathrm{GHz}$. All of

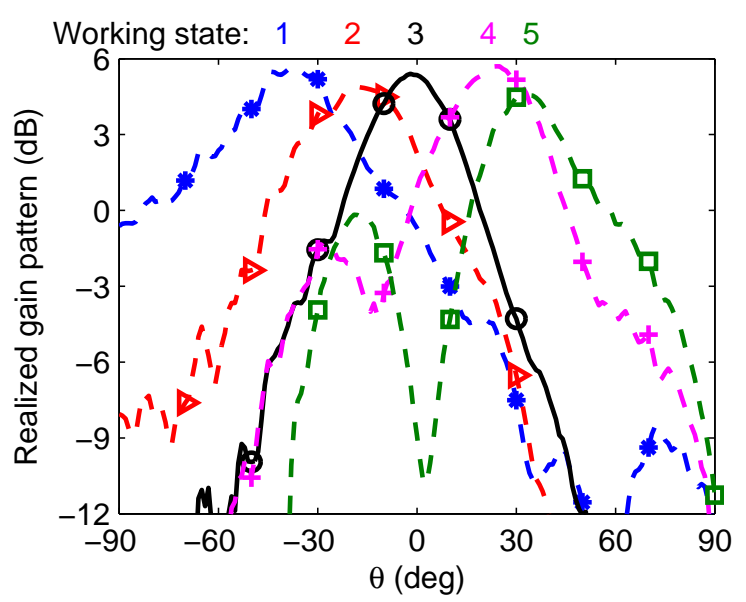

Fig. 19. Measured realized gain patterns for five of the antenna's operating states at $5.0 \mathrm{GHz}$.

TABLE IV

Measured Performance CHARACTERISTics of the FIXED-FREQUENCY BEAM SCANNING CRLH LWA OPERATING AT $5.25 \mathrm{GHz}$

\begin{tabular}{c|cccc}
\hline \hline \multirow{2}{*}{ Antenna performance } & \multicolumn{4}{|c}{ States } \\
\cline { 2 - 5 } & 1 & 2 & 3 & 4 \\
\hline Voltage $V_{1}(\mathrm{~V})$ & 18 & 18 & 18 & 3.1 \\
\hline Voltage $V_{2}(\mathrm{~V})$ & 20 & 16 & 13 & 2 \\
\hline$S_{11}(\mathrm{~dB})$ & -27.5 & -9.6 & -15.9 & -16.5 \\
\hline$S_{21}(\mathrm{~dB})$ & -43.7 & -37.1 & -45.7 & -19.0 \\
\hline Main beam angle & $-15^{\circ}$ & $0^{\circ}$ & $+13^{\circ}$ & $+34^{\circ}$ \\
\hline Realized gain $(\mathrm{dBi})$ & 5.0 & 6.4 & 4.5 & 6.1 \\
\hline \hline
\end{tabular}

the measured $\left|S_{11}\right|$ values are below $-9.6 \mathrm{~dB}$, and those of $\left|S_{21}\right|$ are below $-18.3 \mathrm{~dB}$. The measured radiation patterns for those five working states are depicted in Fig. 19. The measured beam scanning range is from $-37^{\circ}$ to $+32^{\circ}$ when $\left(V_{1}, V_{2}\right)$ is varied from $(18 \mathrm{~V}, 16 \mathrm{~V})$ to $(2.4 \mathrm{~V}, 0.1 \mathrm{~V})$. When $\left(V_{1}, V_{2}\right)=(10.5 \mathrm{~V}, 11 \mathrm{~V})$, the system is in State 3 and the measured main beam points in the broadside direction with the peak realized gain, $5.4 \mathrm{dBi}$. It is found that the half-power beamwidth (HPBW) of the measured realized gain patterns is slightly larger in comparison to the simulated value. Note that the maximum forward beam angle $\left(+32^{\circ}\right)$ of the measured results for State 5 is smaller than the simulated State 6 value, $+62^{\circ}$. As discussed, the missing measured state six arises from the limited capacitance tuning values $C_{V 2}$ associated with the SMV1405 varactors, i.e., the required maximum value of $C_{V 2}$ is $4.0 \mathrm{pF}$, whereas the practical maximum value is only 2.67 $\mathrm{pF}$.

Table IV gives the measured performance of the fixedfrequency beam scanning CRLH LWA in its four working states at $5.25 \mathrm{GHz}$. The measured $\left|S_{11}\right|$ values are below -9.6 $\mathrm{dB}$, and those of $\left|S_{21}\right|$ are below $-19.0 \mathrm{~dB}$. The measured patterns in those four working states are shown in Fig. 20. The measured beam scans from $-15^{\circ}$ to $+34^{\circ}$ when $\left(V_{1}, V_{2}\right)$ 


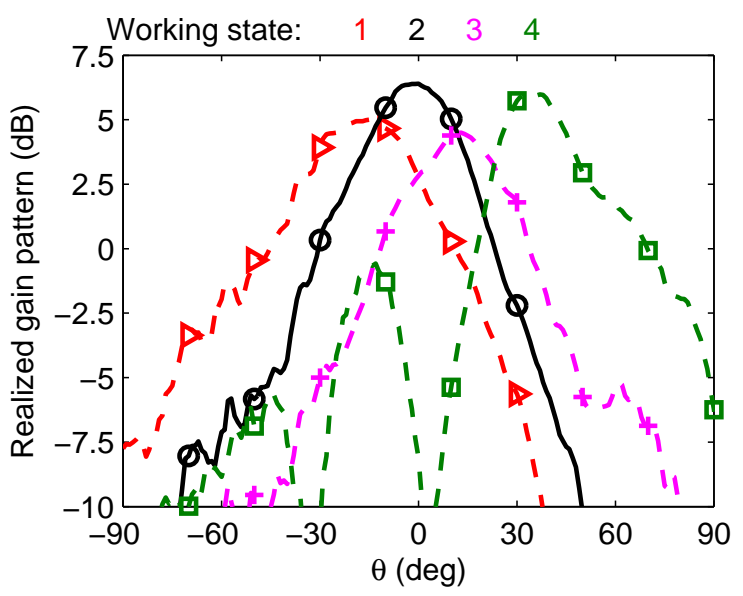

Fig. 20. Measured realized gain patterns for four of the antennas operating states at $5.25 \mathrm{GHz}$.

TABLE V

Realized Gain Values for the Six Operating States With DifFERENT VALUES OF $R_{V 1}$ AND $R_{V 2}$

\begin{tabular}{c|c|cccccc}
\hline \hline \multirow{2}{*}{$\begin{array}{c}\text { Resistor values } \\
\left(R_{V 1}, R_{V 2}\right)\end{array}$} & $\begin{array}{c}\text { Antenna } \\
\text { performance }\end{array}$ & 1 & 2 & 3 & 4 & 5 & 6 \\
\cline { 3 - 8 }$(0 \Omega, 0 \Omega)$ & RG (dBi) & 7.2 & 6.9 & 8.3 & 9.7 & 9.3 & 7.2 \\
\cline { 2 - 8 } & Beam angle & $-46^{\circ}$ & $-24^{\circ}$ & $0^{\circ}$ & $+14^{\circ}$ & $+34^{\circ}$ & $+62^{\circ}$ \\
\hline \multirow{2}{*}{$(0.5 \Omega, 0.2 \Omega)$} & $\mathrm{RG}(\mathrm{dBi})$ & 6.1 & 5.8 & 7.8 & 8.3 & 8.0 & 5.8 \\
\cline { 2 - 8 } & Beam angle & $-42^{\circ}$ & $-22^{\circ}$ & $4^{\circ}$ & $+16^{\circ}$ & $+36^{\circ}$ & $+62^{\circ}$ \\
\hline \multirow{2}{*}{$(1 \Omega, 0.4 \Omega)$} & $\mathrm{RG}(\mathrm{dBi})$ & 5.7 & 5.4 & 7.7 & 8.1 & 7.5 & 5.2 \\
\cline { 2 - 8 } & Beam angle & $-42^{\circ}$ & $-22^{\circ}$ & $4^{\circ}$ & $+16^{\circ}$ & $+36^{\circ}$ & $+62^{\circ}$ \\
\hline \multirow{2}{*}{$(2 \Omega, 0.8 \Omega)$} & $\mathrm{RG}(\mathrm{dBi})$ & 4.7 & 4.6 & 7.1 & 7.4 & 6.4 & 4.5 \\
\cline { 2 - 8 } & Beam angle & $-40^{\circ}$ & $-20^{\circ}$ & $4^{\circ}$ & $+16^{\circ}$ & $+36^{\circ}$ & $+62^{\circ}$ \\
\hline \hline
\end{tabular}

*RG denotes Realized gain

is varied from $(18 \mathrm{~V}, 20 \mathrm{~V})$ to $(3.1 \mathrm{~V}, 2 \mathrm{~V})$. It points in the broadside in State 2 with the peak realized gain value, 6.4 $\mathrm{dBi}$. The reduced measured scanning range is again due to the limitations of the practical tuning capacitance values of both $C_{V 1}$ and $C_{V 2}$.

\section{Discussion and Comparison}

One observes that the measured realized gains are lower than their simulated values. The maximum gain difference is around $4.0 \mathrm{~dB}$. It is also noticed that the measured $\left|S_{21}\right|$ values are lower than the simulated ones, especially for the forward main beam angles. As anticipated, these outcomes occur because lossless capacitances $C_{V 1}$ and $C_{V 2}$ were used for the simulations. The resistance associated with each varactor was not included. To improve the future predictive capability associated with the diode models, the simulated realized gain and beam angles were obtained for different loss values of the varactor diodes A and B. The loss was included in the HFSS simulations by incorporating a resistor in series with each lumped element capacitor representing the varactor diodes $\mathrm{A}$ and B. In particular, diode A (B) is represented by a resistor $R_{V 1}\left(R_{V 2}\right)$ in series with the capacitance $C_{V 1}\left(C_{V 2}\right)$.

Table $\mathrm{V}$ gives the realized gain values for the six operating states at $5.0 \mathrm{GHz}$ for different combinations of $R_{V 1}$ and $R_{V 2}$. The realized gains obtained when $\left(R_{V 1}, R_{V 2}\right)=(0 \Omega, 0 \Omega)$ denote the lossless case. Table $\mathrm{V}$ indicates that when $\left(R_{V 1}\right.$, $\left.R_{V 2}\right)$ is varied from $(0 \Omega, 0 \Omega)$ to $(2 \Omega, 0.8 \Omega)$, the beam angle shift is less than $6^{\circ}$ for all working states and the largest gain variation is around $2.9 \mathrm{~dB}$. Thus, the variations of the resistors confirm both the anticipated non-trivial decreases in the realized gains and the small differences in the main beam angles. For States 1 and 2, the measured realized gains, 5.7 and $4.9 \mathrm{dBi}$, respectively, suggest that the equivalent $\left(R_{V 1}\right.$, $\left.R_{V 2}\right)$ values are most likely to be $(0.5 \Omega, 0.2 \Omega)$. On the other hand, for States 3 to 5 , the measured gains are 5.4, 5.7 , and $4.6 \mathrm{dBi}$, respectively. The equivalent $\left(R_{V 1}, R_{V 2}\right)$ value is most likely $(2 \Omega, 0.8 \Omega)$. These outcomes confirm the fact that the resistor values of the commercial varactor diodes change with their biasing voltages. Obviously, this feature further complicates any precise modeling effort. Nonetheless, the lossless simulation results proved to be an effective guide to realize the prototype and to anticipate qualitatively its measured performance characteristics.

A comparison between the fixed-frequency continuous beam scanning LWA developed in this paper and the previously reported ones is shown in Table VI. Although several of those structures have realized fixed-frequency continuous beam scanning, most of them are operating only at a single frequency, and the beam scanning range is less than $45^{\circ}$. The antenna developed in [26] operates from $5.5-5.8 \mathrm{GHz}$, but the scanning range is limited and the measured gain variation is over $4 \mathrm{dBi}$. The CRLH LWA reported in [31] achieved a $66^{\circ}$ beam scanning range with consistent, attractive gain values. Nevertheless, its beam scanning characteristics were demonstrated by using several prototypes with different fixed capacitor values rather than, as reported in this work, a single one that employed switching devices with realizable biasing lines. The fixed-frequency beam scanning LWA developed herein was facilitated with varactor diodes biased with simple, practical circuits. The simulated results indicate that it can achieve more than $100^{\circ}$ fixed-frequency beam scan over the range from 4.75 to $5.25 \mathrm{GHz}$. The measured results at 5.0 and $5.25 \mathrm{GHz}$ have a reduced beam scanning range, the prototype being limited by the actual capacitance tuning range associated with the commercially-obtained varactor diodes. The measured beam scanning range would have been wider if the commercially-obtained varactor diodes had provided the same capacitance tuning range as the one employed in the simulations. In principle one could use two varactors in parallel to extend the tuning range. However, given the losses associated with the varactors which would have affected the maximum realized gain, a smaller number of them was preferred for the prototype.

\section{CONCLUSION}

A simple CRLH LWA with practical DC biasing lines was demonstrated that achieved fixed-frequency continuous beam 
TABLE VI

Comparison Between the Fixed-Frequency Continuous Beam Scanning LWA Developed in This Paper and the Previously Reported ONES

\begin{tabular}{|c|c|c|c|c|c|c|}
\hline Reference antenna & Antenna type & $\begin{array}{c}\text { Operating frequencies } \\
(\mathrm{GHz})\end{array}$ & $\begin{array}{c}\text { Antenna length } \\
\left(\lambda_{0}\right)\end{array}$ & $\begin{array}{c}\text { Beam scanning range } \\
\text { (Deg) }\end{array}$ & $\begin{array}{c}\text { Maximum gain } \\
(\mathrm{dB})\end{array}$ & $\begin{array}{c}\text { Gain variation } \\
\text { (dB) }\end{array}$ \\
\hline$[26]$ & Corrugated microstrip line & $5.5-5.8$ & 6.2 & $\simeq 45$ & 8.0 & 4.2 \\
\hline [30] & Microstrip CRLH & 3.23 & 3.0 & -10 to +7.5 & -5.6 & 0.5 \\
\hline$[31]$ & Microstrip CRLH & 6.5 & 3.25 & -31 to +35 & 9.9 & 0.4 \\
\hline [32] & Liquid crystal CRLH & 12.4 & 1.2 & -13 to +18 & Not given & Not given \\
\hline Our simulated results (lossless) & Microstrip CRLH & $4.75-5.25$ & 2.6 & $\geq 100$ & 10.5 & 4.4 \\
\hline \multirow{2}{*}{ Our measured results } & \multirow{2}{*}{ Microstrip CRLH } & 5 & 2.6 & -37 to +32 & 5.7 & 1.3 \\
\hline & & 5.25 & 2.6 & -15 to +34 & 6.4 & 1.9 \\
\hline
\end{tabular}

scanning in a wide operating band. A theoretical model and numerical simulations predicted that practical fixed wide-angle continuous beam scanning could be realized by simultaneously controlling both the left-handed capacitance and inductance in its CRLH unit cells. A numerically optimized single-layer frequency-based CRLH LWA was developed that scanned the main beam from $-58^{\circ}$ to $+47^{\circ}$ with the gain variation of only $2.6 \mathrm{dBi}$, between 8.2 and $10.8 \mathrm{dBi}$, when the source frequency was swept from 4 to $6.5 \mathrm{GHz}$. By developing a means to incorporate a simple yet effective set of DC biasing lines into the design, it was demonstrated that the resulting optimized reconfigurable antenna was able to scan its main beam in an angular range larger than $100^{\circ}$ over a wide frequency band from 4.75 to $5.25 \mathrm{GHz}$. A prototype was fabricated and tested. The conducted measurements verified the predicted functionality of the antenna. The measured results at 5.0 and $5.25 \mathrm{GHz}$ confirmed that the antenna could continuously scan its main beam through broadside from $-37^{\circ}$ to $+32^{\circ}$, and from $-15^{\circ}$ to $+34^{\circ}$, respectively. Because of the limitations of the actual capacitance tuning range associated with the commercially-obtained varactor diodes, the measured scanning range was reduced from that predicted by the simulations. Nonetheless, the measured results confirmed that a fixedfrequency beam scanning LWA was achieved. Because its unit cell design and DC biasing network are simple, the realized LWA prototype is a promising candidate for future wireless communication systems that require fixed-frequency beam scanning over a large range of frequencies.

\section{REFERENCES}

[1] D. R. Jackson, C. Caloz, and T. Itoh, "Leaky-wave antennas," Proc. IEEE, vol. 100, no. 7, pp. 2194-2206, Jul. 2012.

[2] C. Jin and A. Alphones, "Leaky-wave radiation behavior from a double periodic composite right/left-handed substrate integrated waveguide," IEEE Trans. Antennas Propag., vol. 60, no. 4, pp. 1727-1735, Apr. 2012.

[3] J. Liu, D. R. Jackson, Y. Li, C. Zhang, and Y. Long, "Investigations of SIW leaky-wave antenna for endfire-radiation with narrow beam and sidelobe suppression," IEEE Trans. Antennas Propag., vol. 62, no. 9, pp. 4489-4497, Sep. 2014.

[4] Z. Li, J. Wang, Z. Zhang, and M. Chen, "Far field computation of the traveling wave structures and a new approach for suppressing the sidelobe levels," IEEE Trans. Antennas Propag., vol. 61, no. 4, pp. 23082312, Apr. 2013.
[5] H. Lee, J. H. Choi, C. M. Wu, and T. Itoh, "A compact single radiator CRLH-inspired circularly polarized leaky-wave antenna based on substrate-integrated waveguide," IEEE Trans. Antennas Propag., vol. 63 , no. 10 , pp. $4566-4572$, Oct. 2015.

[6] N. Nguyen-Trong, L. Hall, and C. Fumeaux, "Transmission-line model of nonuniform leaky-wave antennas," IEEE Trans. Antennas Propag., vol. 64, no. 3, pp. 883-893, Mar. 2016.

[7] Y. Geng, J. Wang, Y. Li, Z. Li, M. Chen, and Z. Zhang, "Leakywave antenna array with a power-recycling feeding network for radiation efficiency improvement," IEEE Trans. Antennas Propag., vol. 65, no. 5, pp. 2689-2694, May 2017.

[8] S. Paulotto, P. Baccarelli, F. Frezza, and D. R. Jackson, "Full-wave modal dispersion analysis and broadside optimization for a class of microstrip CRLH leaky-wave antennas," IEEE Trans. Microw. Theory Tech., vol. 56, no. 12, pp. 2826-2837, Dec. 2008.

[9] Y. Li, Q. Xue, E. K.-N. Yung, and Y. Long, "The periodic half-width microstrip leaky-wave antenna with a backward to forward scanning capability," IEEE Trans. Antennas Propag., vol. 58, no. 3, pp. 963-966, Mar. 2010.

[10] D. K. Karmokar and K. P. Esselle, "Periodic U-slot-loaded dual-band half-width microstrip leaky-wave antennas for forward and backward beam scanning," IEEE Trans. Antennas Propag., vol. 63, no. 12, pp. 5372-5381, Dec. 2015.

[11] A. Lai, T. Itoh, and C. Caloz, "Composite right/left-handed transmission line metamaterials," IEEE Microwave Mag., vol. 5, no. 3, pp. 34-50, Sep. 2004.

[12] G. V. Eleftheriades, A. K. Iyer, and P. C. Kremer, "Planar negative refractive index media using periodically L-C loaded transmission lines," IEEE Trans. Microw. Theory Tech., vol. 50, no. 12, pp. 2702-2712, Dec 2002.

[13] Nasimuddin, Z. N. Chen, and X. Qing, "Multilayered composite right/left-handed leaky-wave antenna with consistent gain," IEEE Trans. Antennas Propag., vol. 60, no. 11, pp. 5056-5062, Nov. 2012.

[14] — "Substrate integrated metamaterial-based leaky-wave antenna with improved boresight radiation bandwidth," IEEE Trans. Antennas Propag., vol. 61, no. 7, pp. 3451-3457, Jul. 2013.

[15] Y. L. Lyu, X. X. Liu, P. Y. Wang, D. Erni, Q. Wu, C. Wang, N. Y. Kim, and F. Y. Meng, "Leaky-wave antennas based on noncutoff substrate integrated waveguide supporting beam scanning from backward to forward," IEEE Trans. Antennas Propag., vol. 64, no. 6, pp. 2155-2164, Jun. 2016.

[16] J. T. Williams, P. Baccarelli, S. Paulotto, and D. R. Jackson, "1-D combline leaky-wave antenna with the open-stopband suppressed: Design considerations and comparisons with measurements," IEEE Trans. Antennas Propag., vol. 61, no. 9, pp. 4484-4492, Sep. 2013.

[17] D. K. Karmokar, Y. J. Guo, P. Y. Qin, S. L. Chen, and T. S. Bird, "Substrate integrated waveguide-based periodic backward-to-forward scanning leaky-wave antenna with low cross-polarization," IEEE Trans. Antennas Propag., vol. 66, no. 8, pp. 3846-3856, Aug. 2018.

[18] S. Paulotto, P. Baccarelli, F. Frezza, and D. R. Jackson, "A novel technique for open-stopband suppression in 1-D periodic printed leakywave antennas," IEEE Trans. Antennas Propag., vol. 57, no. 7, pp. 18941906, Jul. 2009.

[19] Y. L. Lyu, F. Y. Meng, G. H. Yang, Q. Wu, and K. Wu, "Leaky-wave antenna with alternately loaded complementary radiation elements," 
IEEE Antennas Wireless Propag. Lett., vol. 17, no. 4, pp. 679-683, Apr. 2018.

[20] R. O. Ouedraogo, E. J. Rothwell, and B. J. Greetis, "A reconfigurable microstrip leaky-wave antenna with a broadly steerable beam," IEEE Trans. Antennas Propag., vol. 59, no. 8, pp. 3080-3083, Aug. 2011.

[21] R. Guzman-Quiros, J. L. Gomez-Tornero, A. R. Weily, and Y. J. Guo, "Electronically steerable 1-D Fabry-Perot leaky-wave antenna employing a tunable high impedance surface," IEEE Trans. Antennas Propag., vol. 60, no. 11, pp. 5046-5055, Nov. 2012.

[22] D. K. Karmokar, K. P. Esselle, and S. G. Hay, "Fixed-frequency beam steering of microstrip leaky-wave antennas using binary switches," IEEE Trans. Antennas Propag., vol. 64, no. 6, pp. 2146-2154, Jun. 2016.

[23] K. Zhang, H. Yu, X. Ding, and Q. Wu, "Experimental validation of active holographic metasurface for electrically beam steering," Opt. Express, vol. 26, no. 5, pp. 6316-6324, Mar. 2018.

[24] J. M. Montero, A. M. Ocampo, and N. J. G. Fonseca, "C-band multiple beam antennas for communication satellites," IEEE Trans. Antennas Propag., vol. 63, no. 4, pp. 1263-1275, Apr. 2015.

[25] R. Guzman-Quiros, J. L. Gomez-Tornero, A. R. Weily, and Y. J. Guo, "Electronic full-space scanning with 1-D Fabry-Prot LWA using electromagnetic band-gap," IEEE Antennas Wireless Propag. Lett., vol. 11, pp. 1426-1429, 2012.

[26] M. Wang, H. F. Ma, H. C. Zhang, W. X. Tang, X. R. Zhang, and T. J. Cui, "Frequency-fixed beam-scanning leaky-wave antenna using electronically controllable corrugated microstrip line," IEEE Trans. Antennas Propag., vol. 66, no. 9, pp. 4449-4457, Sep. 2018.

[27] L. Chang, Y. Li, Z. Zhang, and Z. Feng, "Reconfigurable 2-bit fixedfrequency beam steering array based on microstrip line," IEEE Trans. Antennas Propag., vol. 66, no. 2, pp. 683-691, Feb. 2018.

[28] L. Ji, Y. J. Guo, P. Qin, S. Gong, and R. Mittra, "A reconfigurable partially reflective surface (prs) antenna for beam steering," IEEE Trans. Antennas Propag., vol. 63, no. 6, pp. 2387-2395, Jun. 2015.

[29] J. Li, M. He, C. Wu, and C. Zhang, "Radiation-pattern-reconfigurable graphene leaky-wave antenna at terahertz band based on dielectric grating structure," IEEE Antennas Wireless Propag. Lett., vol. 16, pp. 1771-1775, 2017

[30] S. Lim, C. Caloz, and T. Itoh, "Electronically scanned composite right/left handed microstrip leaky-wave antenna," IEEE Microw. Wireless Compon. Lett., vol. 14, no. 6, pp. 277-279, Jun. 2004.

[31] A. Suntives and S. V. Hum, "A fixed-frequency beam-steerable halfmode substrate integrated waveguide leaky-wave antenna," IEEE Trans. Antennas Propag., vol. 60, no. 5, pp. 2540-2544, May 2012.

[32] B. Che, T. Jin, D. Erni, F. Meng, Y. Lyu, and Q. Wu, "Electrically controllable composite right/left-handed leaky-wave antenna using liquid crystals in PCB technology," IEEE Trans. Compon. Packag. Manuf. Technol., vol. 7, no. 8, pp. 1331-1342, Aug. 2017.

[33] K. Chen, Y. H. Zhang, S. Y. He, H. T. Chen, and G. Q. Zhu, "An electronically controlled leaky-wave antenna based on corrugated SIW structure with fixed-frequency beam scanning," IEEE Antennas Wireless Propag. Lett., vol. 18, no. 3, pp. 551-555, Mar. 2019.

[34] C. Caloz and T. Itoh, Electromagnetic Metamaterials: Transmission Line Theory and Microwave Applications. John Wiley \& Sons, 2005.

[35] G. V. Eleftheriades and K. G. Balmain, Negative-Refraction Metamaterials: Fundamental Principles and Applications. John Wiley \& Sons, 2005.

[36] N. Engheta and R. W. Ziolkowski, Metamaterials: Physics and Engineering Explorations. John Wiley \& Sons, 2006

[37] Y. Dong and T. Itoh, "Metamaterial-based antennas," Proc. IEEE, vol. 100, no. 7, pp. 2271-2285, Jul. 2012.

[38] D. R. Jackson and A. A. Oliner, "Leaky-Wave Antennas," in Modern Antenna Handbook, C. A. Balanis, Ed. New York: Wiley, 2008, ch. 7.

[39] Y. J. Cheng, W. Hong, K. Wu, and Y. Fan, "Millimeter-wave substrate integrated waveguide long slot leaky-wave antennas and two-dimensional multibeam applications," IEEE Trans. Antennas Propag., vol. 59, no. 1, pp. 40-47, Jan. 2011 .

[40] H. Mosallaei and K. Sarabandi, "Design and modeling of patch antenna printed on magneto-dielectric embedded-circuit metasubstrate," IEEE Trans. Antennas Propag., vol. 55, no. 1, pp. 45-52, Jan. 2007.

[41] H. W. Johnson and M. Graham, High-Speed Digital Design: a Handbook of Black Magic. Englewood Cliffs, NJ: Prentice Hall, 1993.

[42] D. Comite, S. K. Podilchak, P. Baccarelli, P. Burghignoli, A. Galli, A. P. Freundorfer, and Y. M. M. Antar, "A dual-layer planar leakywave antenna designed for linear scanning through broadside," IEEE Antennas Wireless Propag. Lett., vol. 16, pp. 1106-1110, 2017.

[43] J. Liu, W. Zhou, and Y. Long, "A simple technique for open-stopband suppression in periodic leaky-wave antennas using two nonidentical elements per unit cell," IEEE Trans. Antennas Propag., vol. 66, no. 6, pp. 2741-2751, Jun. 2018

[44] N. Nguyen-Trong, L. Hall, and C. Fumeaux, "A frequency- and patternreconfigurable center-shorted microstrip antenna," IEEE Antennas Wireless Propag. Lett., vol. 15, pp. 1955-1958, 2016.

[45] Y. Cai, K. Li, Y. Yin, S. Gao, W. Hu, and L. Zhao, "A low-profile frequency reconfigurable grid-slotted patch antenna," IEEE Access, vol. 6, pp. 36305-36312, 2018.

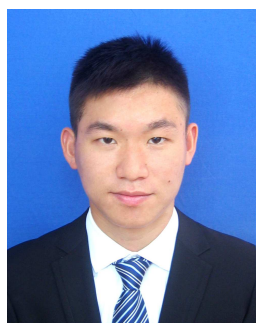

Shu-Lin Chen (S'16) was born in Hubei Province, China. He received the B.S. degree in electrical engineering from Fuzhou University, China, in 2012. and the M.S. degree in electromagnetic field and microwave technology from Xiamen University, China, in 2015. Since 2016, he has been working towards the $\mathrm{PhD}$ degree in Engineering with the Global Big Data Technologies Centre (GBDTC), University of Technology Sydney (UTS), Australia.

His research interests include reconfigurable antennas, leaky-wave antennas, millimeter wave antennas, and adaptive array processing. He was a finalist of ISAP 2017 best paper competition, and his paper was listed as an Honorary Mention in APS-URSI 2017.

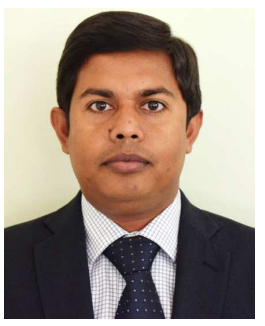

Debabrata K. Karmokar (S'12-M'15) received the B.Sc. degree in electrical \& electronic engineering (EEE) from the Khulna University of Engineering \& Technology (KUET), Khulna, Bangladesh, in 2007 and the PhD degree in Electronic Engineering from Macquarie University, Sydney, NSW, Australia in 2015. He was an Assistant Professor and a member of CRTS in the department of EEE, KUET, and an Assistant Director of Students' Welfare of the University. Between 2012 and 2015, he was a Research Assistant, a Casual Academic, and also a Secretary of the IEEE Student Branch, at Macquarie University. From 2016 to 2019 he was a Postdoctoral Research Associate with the Global Big Data Technologies Centre (GBDTC), University of Technology Sydney (UTS), Australia. He is currently a Lecturer with the School of Engineering, Macquarie University, Australia.

Dr. Karmokar is a recipient of several scholarships and awards including a District Council Scholarship from Satkhira District Council, Bangladesh, Technical Scholarship from KUET, a Commonwealth-funded International Postgraduate Research Scholarship (IPRS) together with an International Macquarie University Research Excellence Scholarship (iMQRES), an OCE $\mathrm{PhD}$ Scholarship from the Commonwealth Scientific and Industrial Research Organisation (CSIRO) ICT Centre, Marsfield, Australia, the First Prize in the Poster Competition at the Engineering Symposium 2015, Macquarie University, Young Scientist Award from Venus International Foundation in 2018, and URSI Young Scientist Award in 2019. He is serving as a reviewer for several journals of IEEE, IET, Wiley, Springer and Elsevier.

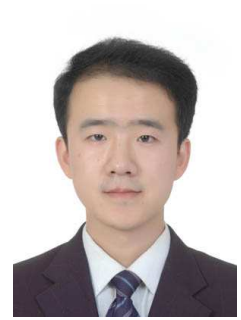

Zheng Li (M'15) received the B.S. degree in physics and $\mathrm{Ph} . \mathrm{D}$. degree in electrical engineering from Beijing Jiaotong University, Beijing, China, in 2006 and 2012, respectively. From 2008 to 2009, he was a visiting student with the Pennsylvania State University, Pennsylvania, USA. From 2017 to 2018, he was a visiting professor with the Global Big Data Technologies Centre, University of Technology Sydney, Australia.

In 2012, he joined the faculty of the Department of Electrical Engineering, Beijing Jiaotong University, where he became an associate professor in 2015. His research interests include leaky-wave antennas and leaky waveguides. 


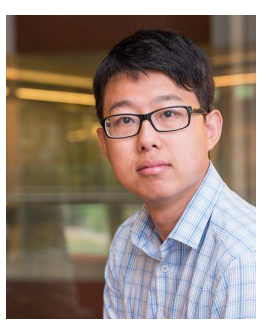

Pei-Yuan Qin (M'13) was born in Liaoning Province, China, in 1983. He received a Bachelor Degree in Electronic Engineering from Xidian University, Xian, China, in 2006, and a joint $\mathrm{Ph} . \mathrm{D}$. Degree from Xidian University and Macquarie University, Australia, in electromagnetic fields and microwave technology in 2012.

From 2012 to 2015, he was a Postdoctoral Research Fellow in Commonwealth Scientific and Industrial Research Organisation (CSIRO), Australia. From 2015 to 2016, he was a Chancellor's Postdoctoral Research Fellow/Lecturer with University of Technology Sydney (UTS), Australia. Since 2017, he is a Senior Lecturer with UTS. His research interests are in the areas of reconfigurable antennas, phase shifters, reconfigurable reflectarrays, and MIMO communications.

Dr. Qin was a recipient of an Australia Research Council Discovery Early Career Researcher Award and also a recipient of the International Macquarie University Research Excellence Scholarship. He was awarded the Vice Chancellors Commendation for academic excellence by Macquarie University. Since 2017, he has been serving as an Associate Editor of the IEEE Antennas and Wireless Propagation Letters.

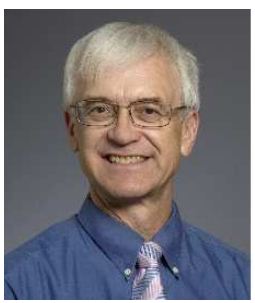

Richard W. Ziolkowski (M'87-SM'91-F'94) received the B.Sc. (magna cum laude) degree (Hons.) in physics from Brown University, Providence, RI, USA, in 1974; the M.S. and Ph.D. degrees in physics from the University of Illinois at UrbanaChampaign, Urbana, IL, USA, in 1975 and 1980, respectively; and the Honorary Doctorate degree from the Technical University of Denmark, Kongens Lyngby, Denmark in 2012.

$\mathrm{He}$ is currently a Distinguished Professor in the Global Big Data Technologies Centre in the Faculty of Engineering and Information Technologies (FEIT) at the University of Technology Sydney, Ultimo NSW, Australia. He became a Professor Emeritus at the University of Arizona in 2018, where he was a Litton Industries John M. Leonis Distinguished Professor in the Department of Electrical and Computer Engineering in the College of Engineering and was also a Professor in the College of Optical Sciences. He was the Computational Electronics and Electromagnetics Thrust Area Leader with the Lawrence Livermore National Laboratory, Engineering Research Division, in Livermore, CA before joining The University of Arizona, Tucson, AZ, USA, in 1990.

Prof. Ziolkowski is the recipient of the 2019 IEEE Electromagnetics Award (IEEE Field Award). He is a Fellow of the Optical Society of America (OSA, 2006), and of the American Physical Society (APS, 2016). He was the Australian DSTO Fulbright Distinguished Chair in Advanced Science and Technology from 2014-2015. He was a 2014 Thomas-Reuters Highly Cited Researcher. He served as the President of the IEEE Antennas and Propagation Society in 2005. He is also actively involved with the URSI, OSA and SPIE professional societies.

His current research interests include the application of new mathematical and numerical methods to linear and nonlinear problems dealing with the interaction of electromagnetic and acoustic waves with complex linear and nonlinear media, as well as metamaterials, metamaterial-inspired structures, nano-structures, and other classical and quantum applications-specific configurations.

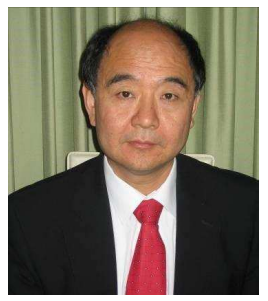

Y. Jay Guo (Fellow'2014) received a Bachelor Degree and a Master Degree from Xidian University in 1982 and 1984, respectively, and a PhD Degree from Xian Jiaotong University in 1987, all in China. His research interest includes antennas, mm-wave and $\mathrm{THz}$ communications and sensing systems as well as big data technologies. He has published over 400 research papers and holds 24 patents in antennas and wireless systems. He is a Fellow of the Australian Academy of Engineering and Technology, a Fellow of IEEE and a Fellow of IET, and a member of the College of Experts of Australian Research Council (ARC). He has won a number of most prestigious Australian national awards, and was named one of the most influential engineers in Australia in 2014 and 2015.

Prof. Guo is a Distinguished Professor and the founding Director of Global Big Data Technologies Centre at the University of Technology Sydney (UTS), Australia. Prior to this appointment in 2014, he served as a Director in CSIRO for over nine years, directing a number of ICT research portfolios. Before joining CSIRO, he held various senior technology leadership positions in Fujitsu, Siemens and NEC in the U.K.

Prof. Guo has chaired numerous international conferences. He is the Chair Elect of International Steering Committee, International Symposium on Antennas and Propagation (ISAP). He was the International Advisory Committee Chair of IEEE VTC2017, General Chair of ISAP2015, iWAT2014 and WPMC'2014, and TPC Chair of 2010 IEEE WCNC, and 2012 and 2007 IEEE ISCIT. He served as Guest Editor of special issues on Antennas for Satellite Communications and Antennas and Propagation Aspects of 6090GHz Wireless Communications, both in IEEE Transactions on Antennas and Propagation, Special Issue on Communications Challenges and Dynamics for Unmanned Autonomous Vehicles, IEEE Journal on Selected Areas in Communications (JSAC), and Special Issue on 5G for Mission Critical Machine Communications, IEEE Network Magazine. 